\title{
A UNIQUENESS CONDITION FOR NONLINEAR CONVECTION-DIFFUSION EQUATIONS WITH DISCONTINUOUS COEFFICIENTS
}

\author{
Stefan Diehl \\ Centre for Mathematical Sciences, Lund University, P.O. Box 118, SE-221 00 Lund, Sweden \\ diehl@maths.lth.se \\ Received (Day Mth. Year) \\ Revised (Day Mth. Year) \\ Communicated by [editor]
}

\begin{abstract}
The paper focuses on the uniqueness issue for scalar convection-diffusion equations where both the convective flux and diffusion functions have a spatial discontinuity. An interface entropy condition is proposed at such a spatial discontinuity. It implies the Kružkov-type entropy condition presented by Karlsen et al. [Trans. Royal Norwegian Society Sci. Letters 3, 49 pp, 2003]. They proved uniqueness when the convective flux function satisfies an additional 'crossing condition'. The crossing condition becomes redundant with the entropy condition proposed here. Thereby, more general flux functions are allowed. Another advantage of the entropy condition is its simple geometrical interpretation, which facilitates the construction of stationary solutions.
\end{abstract}

Keywords: degenerate parabolic equation; nonlinear scalar convection-diffusion equation; conservation law; discontinuous coefficient; uniqueness; coupling condition; interface entropy condition.

\section{Introduction}

\subsection{The problem}

We are interested in the uniqueness question for nonlinear strongly degenerate parabolic scalar convection-diffusion initial-value problems of the type

$$
\begin{array}{ll}
u_{t}+\left(F(u, x)-D(u, x)_{x}\right)_{x}=0, & (x, t) \in \Pi_{T}=\mathbb{R} \times(0, T), \\
u(x, 0)=u_{0}(x), & x \in \mathbb{R},
\end{array}
$$

with the discontinuous flux and diffusion functions

$$
F(u, x)=\left\{\begin{array}{ll}
f(u), & x>0, \\
g(u), & x<0,
\end{array} \text { and } D(u, x)= \begin{cases}A(u), & x>0 \\
B(u), & x<0 .\end{cases}\right.
$$

Here, $u=u(x, t) \geq 0$ is the unknown function, and the given data are: $T>0$, $u_{0} \in L^{\infty}(\mathbb{R}), f$ and $g$ are Lipschitz continuous convective flux functions, and $A$ and 
$B$ are non-decreasing Lipschitz continuous diffusion functions satisfying $A(0)=$ $B(0)=0$. Each diffusion function is constant on a finite number of disjoint closed intervals and on the complement $A^{\prime}>0\left(B^{\prime}>0\right)$ holds. When they are constant, Equation (1.1) degenerates to a hyperbolic equation. Consequently, discontinuities may appear in the solution also away from the spatial discontinuity at $x=0$ of the flux and diffusion functions.

\subsection{Applications}

Equations like (1.1) arise in different applications. When $D=0$, Equation (1.1) becomes a first-order hyperbolic model, a conservation law with a discontinuous flux, which appears in the modelling of two-phase flow in heterogeneous media [31,33], traffic flow with abruptly changing surface conditions, or number of lanes, $[9,10,41]$, and continuous sedimentation in a clarifier-thickener unit $[13,14,19,21]$. In these three areas of application it is also natural to consider a second-order model with diffusion present: see $[28,42]$ in the case of two-phase flow, [10] in traffic flow, and [15] in continuous sedimentation.

In particular, we highlight the well-posedness analysis of the idealized model of continuous sedimentation with compression in a clarifier-thickener unit presented by Bürger et al. [15]. The model can be written

$$
u_{t}+f(\gamma(x), u)_{x}=\left(\gamma_{1}(x) A(u)_{x}\right)_{x},
$$

where $\gamma(x)$ is a piecewise constant vector-valued function and $\gamma_{1}(x)$ is the characteristic function of a bounded interval, which corresponds to the total depth of the sedimentation tank. At one inlet and two outlets of the sedimentation tank the flux function is space-discontinuous. There is diffusion present only inside the sedimentation tank. At the three locations of the spatial flux-discontinuities, the diffusion term is either continuous (inlet), or identically zero on one side and not the other (outlets). Equation (1.1) serves as a model of each of these three locations corresponding to $A=B$ (inlet), $B=0$ (outlet at the top) and $A=0$ (outlet at the bottom), respectively. The presence of this space-discontinuous diffusion term has been the motivation for the analysis in the present paper. The results of [15] are based on the analyses by Karlsen et al. [34,35] and Bürger et al. [14].

\subsection{Two entropy concepts}

A general result on uniqueness was obtained by Karlsen et al. [35], who analyzed the initial-value problem for the strongly degenerate parabolic equation

$$
u_{t}+f(\gamma(x), u)_{x}=A(u)_{x x},
$$

where $\gamma(x)$ is a piecewise smooth and bounded vector-valued function with a finite number of discontinuities. A Kružkov-type entropy condition was proposed and uniqueness was shown via $L^{1}$ stability of weak entropy solutions, provided that an additional 'crossing condition' holds. This condition involves the two flux functions 
$f\left(\gamma\left(x_{\mathrm{d}} \pm 0\right), \cdot\right)$, where $x_{\mathrm{d}}$ is a point where $\gamma$ is discontinuous. The key issue in the analysis of (1.3) concerns each such spatial discontinuity of the convective flux function. Ignoring a possible smooth $x$-dependence of $\gamma(x)$ between the spatial discontinuities, we may set $f(u):=f\left(\gamma\left(x_{\mathrm{d}}+0\right), u\right)$ and $g(u):=f\left(\gamma\left(x_{\mathrm{d}}-0\right), u\right)$, and refer to (1.1) with $A=B$ for simplicity of notation and the purpose of the present paper.

The Kružkov-type entropy condition by Karlsen et al. [35] implies partly the standard entropy condition away from the spatial discontinuities (see $\mathrm{Wu}$ and Yin [47] and Evje and Karlsen [29]), partly an interface entropy condition at such discontinuities. It yields the physically relevant solution of the clarifier-thickener (CT) problem for given initial data. Let us call such a solution a clarifier-thickener entropy (CTE) solution to distinguish it from another type of entropy solution commented on below (well aware of the fact that there are several other suitable applications except sedimentation). We note that the uniqueness issue for CTE solutions has been widely studied, mostly in the hyperbolic special case when $D=0$ in (1.1), e.g. [7,14,15,18,19,20,27,30,31,32,35,36,37,38,39,44,45].

In the notation of (1.1), the crossing condition in [35] imposes conditions on $f$ and $g$, for example, that the graphs of $f$ and $g$ may intersect at most at one point. The crossing condition has also been used in $[14,36]$ by Bürger, Karlsen et al. The reason for its presence has been to rule out situations in which their Kružkov-type CTE condition seems not to be sufficiently strong. It is also concluded in [35] that limit solutions of the numerical schemes, used there to prove existence, seem to satisfy some additional entropy condition which is not captured by theirs.

In consequence, Bürger et al. [16] propose another Kružkov-type entropy formulation, which does not require the crossing condition to ensure uniqueness. They treat, for clarity, the hyperbolic case of (1.1) when $D=0$, but comment that the analysis could be generalized to include diffusion. Their interface entropy inequality involves both a problem-specific 'flux connection', introduced by Adimurthi et al. [2], and a single 'adapted Kružkov entropy' of the type used by Audusse and Perthame [6]. Audusse and Perthame introduced an infinite family of adapted entropies and showed uniqueness without an interface condition and without any assumption on the traces of the solution at $x=0$. In a simpler setup, the idea of using adapted entropies goes back to Baiti and Jenssen [8].

The problem-specific flux connection (in [16]) should be defined a priori and it corresponds to a stationary solution, which is either an undercompressive or marginally undercompressive wave at $x=0$. Adimurthi et al. $[1,2,3]$ used such a connection in an interface entropy condition to prove uniqueness for piecewise smooth solutions. The condition implies that the solution contains no undercompressive waves at $x=0$ except possibly the problem-specific flux connection. The condition is physically motivated in two-phase flow in heterogeneous media, see Kaasschieter [33], who derives such a condition in the hyperbolic limit of a parabolic equation in which an application-specific nonlinear diffusion term vanishes. 
With this new flux-connection-adapted-entropy (FCAE) condition at $x=0$ and the standard Kružkov entropy condition ([40]) away from $x=0$, Bürger et al. [16] establish uniqueness and existence (for the hyperbolic case $D=0$ ) and generalize the results in [2].

There are some advantages with the FCAE condition. The crossing condition is not needed and proofs of convergence of numerical algorithms are easier, see [16]. Most important is perhaps the fact that the FCAE solutions seem to be the physically relevant ones for two-phase flow in heterogeneous media, see [3,33], and in the modelling of traffic flow, see [9]. We emphasize that different solutions may be picked by the CTE and the FCAE conditions, see Bürger et al. [12]. The possibility of adapting the entropy condition to different physical problems is then an advantage. However, this has so far only been done for these two physical problems (two-phase flow and traffic flow), where the flux functions are not too complicated.

A disadvantage of the FCAE concept in the corresponding references above is that it rely heavily on the shape of the fluxes. Either $f$ and $g$ should be both strictly monotone or both unimodal. The CT problem is in general more complex where $f$ may have both a local minimum and a local maximum (in the interior of the range of $u$ ). In a recent paper, Adimurthi et al. [4] generalize their previous flux-connection entropy condition to the case of several extrema of $f$ and $g$. A vector of flux connections should be defined a priori and their coupling condition is rather involved. They assume that $f^{\prime}$ and $g^{\prime}$ have opposite signs at each flux intersection and that the fluxes are equal at the endpoints of the range of $u$. Both these assumptions are not satisfied in the general CT problem. Furthermore, the FCAE concept has so far only been presented for the hyperbolic subcase when $D=0$ in (1.1).

Another feature of the CT problem is that the fluxes $f$ and $g$ vary with time via volume fluxes and a feed inlet. Furthermore, these fluxes are often used as control variables, which means that they depend on the solution, see $[22,23,24,25,26]$. This suggests that if the FCAE concept should be adapted to the CT problem, the flux connection should depend on the solution instead of being defined a priori. Today it is not clear how this should be done. The CTE concept handles such situations automatically. Therefore, further development of the CTE concept is of interest.

\subsection{The present entropy condition}

This paper deals with the CTE concept. We present an interface entropy condition at $x=0$ for (1.1), called Condition $\Gamma$, which implies uniqueness without the crossing condition. Thereby, more general flux functions are allowed, for example, with several flux crossings. The condition is a reformulation and extension of the one in [18], in which the hyperbolic case $(D=0)$ was treated. It implies the Kružkovtype CTE condition used in [35]. Consequently, when the fluxes $f$ and $g$ satisfy the crossing condition, Condition $\Gamma$ and the Kružkov-type CTE condition in [35] pick the same solution. 
The difference between the two entropy conditions is the following. Condition $\Gamma$ utilizes more specific information of the solution at time $t$ and the flux functions. Firstly, the crossing condition is an a priori condition on the flux functions with no connection to the actual solution. Secondly, given a solution at time $t$ with the traces $u_{ \pm}=u(0 \pm, t)$, the Kružkov-type CTE condition (in [35]) imposes restrictions on both $f$ and $g$ on the whole interval between $u_{+}$and $u_{-}$; denote this closed interval by $\operatorname{ch}\left(u_{+}, u_{-}\right)$. This is unnecessarily strong, since some parts of the fluxes within this interval may be altered arbitrarily without influencing the solution at all. Condition $\Gamma$, on the other hand, imposes restrictions on $f$ only in a closed subinterval $\operatorname{ch}\left(u_{+}, \bar{u}\right) \subset \operatorname{ch}\left(u_{+}, u_{-}\right)$and on $g$ on the subinterval $\operatorname{ch}\left(\bar{u}, u_{-}\right) \subset \operatorname{ch}\left(u_{+}, u_{-}\right)$. Conversely, within these subintervals the fluxes cannot be altered arbitrarily without violating the solution. In this sense, Condition $\Gamma$ is also a necessary condition for uniqueness for CTE solutions. In the hyperbolic case this is supported by the viscous profile analyses in $[20,27]$. Furthermore, the geometrical interpretation of Condition $\Gamma$ is more direct than the Kružkov-type condition in [35]. This makes the construction of stationary solutions easier, see the last section here and the stationary solutions in $[15,17]$ by Bürger et al.

In [5], Aguilar et al. considered the case of (1.1) where the flux function has no spatial discontinuity $(f=g)$ but there is a discontinuity in the diffusion function corresponding to $A=0$ and $B^{\prime}>0$. Their coupling condition at $x=0,[5$, Eq. (3.13)], is a special case of Condition $\Gamma$.

In this paper, we do not treat the existence problem, but merely assume that there exists a weak entropy solution of (1.1) (in a way that is specified below). In order to prove existence, it would probably be needed to reformulate Condition $\Gamma$ to an integral-type entropy inequality. At the moment it is not clear how this should be done. For the equations that are treated by Bürger, Karlsen et al. in $[11,14,15,34,35,36]$, the proofs of existence are carried out by showing convergence of different numerical methods. We refer to those papers for substantial reviews and references of the previous results on uniqueness, existence and numerical methods of either different subcases of (1.1) (such as the hyperbolic case $D=0$, or $A=B$ with or without convexity conditions on $f$ and $g$ ) or cases when there is an additional smooth $x$-dependence of the convective flux besides a finite number of $x$-discontinuities.

The proof of uniqueness of solutions for the clarifier-thickener model (1.2) by Bürger et al. [15] follows closely the one in [35] and required only small modifications. With similar modifications, the proof also covers the slightly more general situation (1.1) if the crossing condition is satisfied. Note that the crossing condition is indeed satisfied in the CT problem (1.2), see [14].

In Section 2, the assumptions, Condition $\Gamma$ and the main theorem are stated. In [18], Condition $\Gamma$ was presented for the hyperbolic case $(D=0)$ in a more involved way. It is reviewed and reformulated as entropy inequalities in Section 3. In Section 4 it is extended to include the degenerate parabolic case. Some immediate properties are stated together with the fact that it implies the Kružkov-type CTE inequality 
used in $[15,35]$. The proof of uniqueness can be found in Section 5 . It follows closely the one in [35]. Modifications are done mainly where the crossing condition and their coupling condition were used, but also to cope with the assumption that $u \in L^{\infty}$ instead of $L^{1}$ (which is used in [15,35]). This is because we are interested in solutions that do not tend to zero far away, which is natural because of the nonlinear flux functions. The simple geometrical interpretation of Condition $\Gamma$ facilitates the construction of stationary solutions. Such examples are given in Section 6, where a monotonicity property of the solutions can also be found.

\section{Weak entropy solution and $L^{1}$ stability}

Definition 2.1 (weak solution). A function $u: \Pi_{T} \rightarrow \mathbb{R}$ is called a weak solution of (1.1) if it satisfies the following assumptions:

(A.1) $u \in L^{\infty}\left(\Pi_{T}\right), A(u)_{x} \in L^{\infty}((0, \infty) \times(0, T))$ and $B(u)_{x} \in L^{\infty}((-\infty, 0) \times$ $(0, T))$.

(A.2) For a.e. $t \in(0, T), A(u(\cdot, t)), B(u(\cdot, t)) \in C(\mathbb{R})$.

(A.3) For all test functions $\varphi \in C_{0}^{\infty}\left(\Pi_{T}\right)$,

$$
\iint_{\Pi_{T}}\left(u \varphi_{t}+\left(F(u, x)-D(u, x)_{x}\right) \varphi_{x}\right) d x d t=0 .
$$

(A.4) The initial condition is satisfies in the following sense, for any $r>0$ :

$$
\lim _{\tau \searrow 0} \frac{1}{\tau} \int_{0}^{\tau} \int_{-r}^{r}\left|u(x, t)-u_{0}(x)\right| d x d t=0 .
$$

(A.5) The following traces exist for a.e. $t \in(0, T)$ :

$$
\begin{aligned}
u_{ \pm} & =u_{ \pm}(t):=\underset{\varepsilon \searrow 0}{\operatorname{ess} \lim } u( \pm \varepsilon, t) \in L^{\infty}(0, T), \\
\mathcal{A}^{u}=\mathcal{A}^{u}(t) & :=\left(A(u)_{x}\right)_{+} \in L^{\infty}(0, T), \\
\mathcal{B}^{u}=\mathcal{B}^{u}(t) & :=\left(B(u)_{x}\right)_{-} \in L^{\infty}(0, T) .
\end{aligned}
$$

The assumption $u \in L^{1}$ in $[15,35]$ by Bürger, Karlsen et al. is here relaxed, since we are interested in solutions that may not tend to zero far away. We refer to [35] for discussions and proofs of the existence of traces in (A.5) in special cases of (1.1). Note that the continuity assumption (A.2) includes the fact that both $A(u)$ and $B(u)$ are continuous over the spatial discontinuity. This is also assumed by Bürger et al. [15] and motivated in that paper.

Note that (A.3) implies the Rankine-Hugoniot condition at $x=0$ (cf. [35]):

$$
f\left(u_{+}\right)-\mathcal{A}^{u}=g\left(u_{-}\right)-\mathcal{B}^{u} .
$$

For two real numbers $\alpha$ and $\beta$, we define the closed convex hull of these by

$$
\operatorname{ch}(\alpha, \beta):=[\min (\alpha, \beta), \max (\alpha, \beta)],
$$


and let the sign function be

$$
\operatorname{sign}(\alpha)= \begin{cases}-1, & \alpha<0, \\ 0, & \alpha=0, \\ 1, & \alpha>0 .\end{cases}
$$

Definition 2.2 (entropy solution). A weak solution $u$ of (1.1) is called an entropy solution if the following conditions hold:

(E.1) For any $c \in \mathbb{R}$ and all test functions $0 \leq \varphi \in C_{0}^{\infty}((-\infty, 0) \times(0, T))$,

$$
\iint_{\Pi_{T}}\left(|u-c| \varphi_{t}+\operatorname{sign}(u-c)\left(g(u)-g(c)-B(u)_{x}\right) \varphi_{x}\right) d x d t \geq 0 .
$$

and for any $c \in \mathbb{R}$ and all test functions $0 \leq \varphi \in C_{0}^{\infty}((0, \infty) \times(0, T))$,

$$
\iint_{\Pi_{T}}\left(|u-c| \varphi_{t}+\operatorname{sign}(u-c)\left(f(u)-f(c)-A(u)_{x}\right) \varphi_{x}\right) d x d t \geq 0 .
$$

(E.2) (Condition $\Gamma$ ) For a.e. $t \in(0, T)$ there exists a number $\bar{u} \in \operatorname{ch}\left(u_{-}, u_{+}\right)$ such that the following holds:

$$
\begin{aligned}
& \left(u_{+}-\bar{u}\right)(f(v)-\eta) \geq 0 \quad \forall v \in \operatorname{ch}\left(u_{+}, \bar{u}\right), \\
& \left(\bar{u}-u_{-}\right)(g(v)-\eta) \geq 0 \quad \forall v \in \operatorname{ch}\left(u_{-}, \bar{u}\right), \\
& \text { where } \eta:=f\left(u_{+}\right)-\mathcal{A}^{u}=g\left(u_{-}\right)-\mathcal{B}^{u} .
\end{aligned}
$$

Theorem 2.3 ( $L^{1}$ stability and uniqueness). Let $u$ and $v$ be two weak entropy solutions of (1.1) with the initial data $u_{0}$ and $v_{0}$, respectively. For a.e. $t \in(0, T)$ and any number $N>0$,

$$
\int_{\mathbb{R}}|u(x, t)-v(x, t)| w_{N}(x) d x \leq e^{C t} \int_{\mathbb{R}}\left|u_{0}(x)-v_{0}(x)\right| w_{N}(x) d x,
$$

where

$$
w_{N}(x)= \begin{cases}\frac{1}{1+(x+N)^{2}}, & x \leq-N, \\ 1, & |x| \leq N \\ \frac{1}{1+(x-N)^{2}}, & x \geq N,\end{cases}
$$

and $C>0$ is a finite constant, which is independent of $N$, but depends on the flux and diffusion functions.

The proof can be found in Section 5. For degenerate parabolic equations with smooth coefficients, a similar stability inequality with a weight function can be found in the early paper by Vol'pert and Hudjaev [46]. 


\section{Entropy condition in the hyperbolic case, $D=0$}

This section is not needed for the proof of Theorem 2.3. The purpose is to rewrite the original formulation of Condition $\Gamma$ in [18] to entropy inequalities. It was in [18] presented for the hyperbolic case $(D=0)$ in a more involved way. Thereby, the background to the general formulation (E.2) is given including the interpretation of the number $\bar{u}$.

When there is no diffusion term $(D=0),(1.1)$ becomes the hyperbolic equation with a discontinuous flux function,

$$
u_{t}+[H(x) f(u)+(1-H(x)) g(u)]_{x}=0 .
$$

For this type of equation, the aim of $[18,19]$ was to present a procedure to construct unique piecewise smooth solutions from bounded piecewise smooth and piecewise monotone initial data. An additional complication was a time dependent source term, which can be incorporated into $g$, but we do not take it into account here. The constructed solutions were assumed to stay sufficiently regular: piecewise $C^{1}$ and traces along $x=0$ piecewise monotone. The following traces were also needed:

$$
u^{ \pm}:=\lim _{\varepsilon \searrow 0} u_{ \pm}(t+\varepsilon)
$$

In order to state and reformulate Condition $\Gamma$, we recall some further notation from [18]. Note that the Rankine-Hugoniot condition of (3.1) at $x=0$ is

$$
f\left(u_{+}\right)=g\left(u_{-}\right) .
$$

Definition 3.1. Given $u_{+}, u_{-} \in \mathbb{R}$, define (cf. Figure 1)

$$
\begin{gathered}
\hat{f}\left(u ; u_{+}\right):=\left\{\begin{array}{cc}
\min _{v \in\left[u, u_{+}\right]} f(v), \quad u \leq u_{+}, \\
\max _{v \in\left[u_{+}, u\right]} f(v), \quad u>u_{+},
\end{array}\right. \\
P\left(f ; u_{+}\right):=\left\{u_{+}\right\} \cup\left\{u \in \mathbb{R}: u<u_{+} ; \hat{f}\left(u+\varepsilon ; u_{+}\right)>\hat{f}\left(u ; u_{+}\right) \forall \varepsilon>0\right\} \\
\cup\left\{u: u>u_{+} ; \hat{f}\left(u-\varepsilon ; u_{+}\right)<\hat{f}\left(u ; u_{+}\right) \forall \varepsilon>0\right\}, \\
\check{g}\left(u ; u_{-}\right):=\left\{\begin{array}{cc}
\max _{v \in\left[u, u_{-}\right]} g(v), \quad u \leq u_{-} \\
\min _{v \in\left[u_{-}, u\right]} g(v), \quad u>u_{-}
\end{array}\right\}=\hat{g}\left(u_{-} ; u\right), \\
N\left(g ; u_{-}\right):=\left\{\begin{aligned}
\left.u u_{-}\right\} \cup\left\{u \in \mathbb{R}: u<u_{-} ; \check{g}\left(u+\varepsilon ; u_{-}\right)<\check{g}\left(u ; u_{-}\right) \forall \varepsilon>0\right\} \\
\cup\left\{u: u>u_{-} ; \check{g}\left(u-\varepsilon ; u_{-}\right)>\check{g}\left(u ; u_{-}\right) \forall \varepsilon>0\right\} .
\end{aligned}\right.
\end{gathered}
$$

For the projection on the u-axis of the intersection of the graphs of $\hat{f}$ and $\check{g}$ we define

$$
\bar{U}=\bar{U}\left(u_{+}, u_{-}\right):=\left\{u \in \mathbb{R}: \hat{f}\left(u ; u_{+}\right)=\check{g}\left(u ; u_{-}\right)\right\}
$$

and let $\bar{u} \in \bar{U} \cap \operatorname{ch}\left(u_{-}, u_{+}\right)$denote an arbitrary point. Since $\hat{f}$ is non-decreasing and $\check{g}$ is non-increasing, the set $\bar{U}$ is an interval, which may consist of a single point $\bar{u}$ 

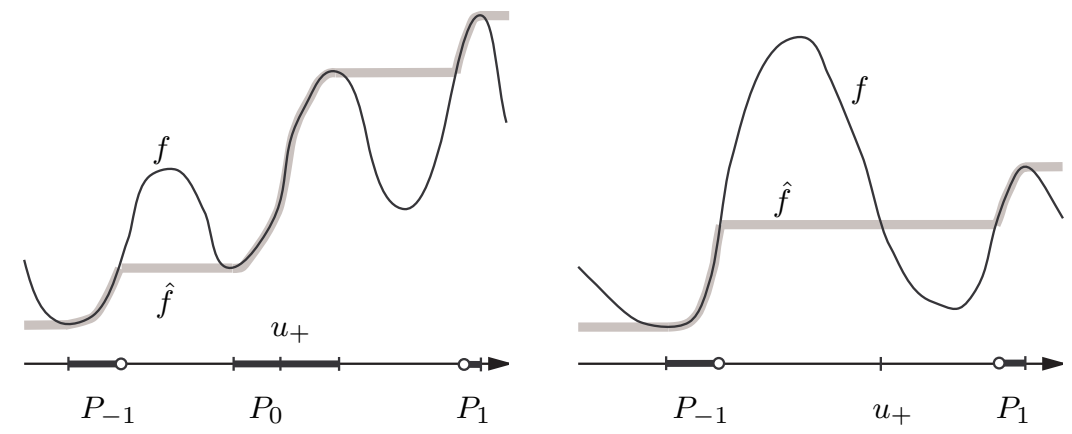

Fig. 1. Examples of graphs of $f$ (solid) and the two main possibilities for $\hat{f}\left(\cdot ; u_{+}\right)$(grey). The set $P=\cup_{i} P_{i}$, where $P_{0}=u_{+}$in the right-hand figure.

(cf. Figures 2-5). Furthermore, introduce the set of pairs

$$
\Gamma\left(u_{+}, u_{-}\right):=\left\{(\alpha, \beta) \in \mathbb{R}^{2}: f(\alpha)=g(\beta)=\hat{f}\left(\bar{U} ; u_{+}\right)\right\} .
$$

Note that, in this section, the number $\bar{u}$ belongs to the set $\bar{U}$, in contrast to the general formulation of Condition $\Gamma$ in Definition 2.2.

The set $\Gamma$ contains generally several pairs that satisfy the Rankine-Hugoniot condition (3.2) and therefore are candidates of 'new' boundary values for the continuation of a (piecewise smooth) solution at $t=0+$. However, it turns out that only one of these pairs are possible for the continuation of a solution. Therefore, the following condition was introduced in [18].

Condition $\Gamma$ (version $1, D=0$, piecewise smooth solutions): $\left(u^{+}, u^{-}\right) \in$ $\Gamma\left(u_{+}, u_{-}\right)$for every $t \in(0, T)$.

A characterization of a solution that satisfies this condition is the following. Firstly, the variation of $u(\cdot, t)$ is minimized. Secondly, if there is more than one pair $\left(u^{+}, u^{-}\right)$with the same variation, then the jump $\left|u^{+}-u^{-}\right|$is minimized, see [18, Examples 2.7 and 2.8]. The order of these minimizations is important, since there need not exist a minimal jump. If interpreting $u^{+}$and $u^{-}$as possible 'inner states' along $x=0$, the minimal jump condition by Gimse and Risebro [30] is equivalent to Condition $\Gamma$.

For the simpler Riemann problem, where the initial data consist of a single step at $x=0$ between two constants $u_{ \pm}$, Condition $\Gamma$ reduces to

$$
\left(u^{+}, u^{-}\right)=c\left(u_{+}, u_{-}\right):=\left(P\left(f ; u_{+}\right) \times N\left(g ; u_{-}\right)\right) \cap \Gamma\left(u_{+}, u_{-}\right),
$$

where the set on the right hand side consists of precisely one member (since the restrictions $\left.f\right|_{P}$ and $\left.g\right|_{N}$ are injective, see [18, Proposition 2.11]). Hence, the coupling function $c$ is well-defined. For the construction of solutions, locally in time, when the initial-value function is piecewise smooth and piecewise monotone, the procedure in [18] utilizes the coupling function $c$ twice. For such regular solutions, the fixed-point 

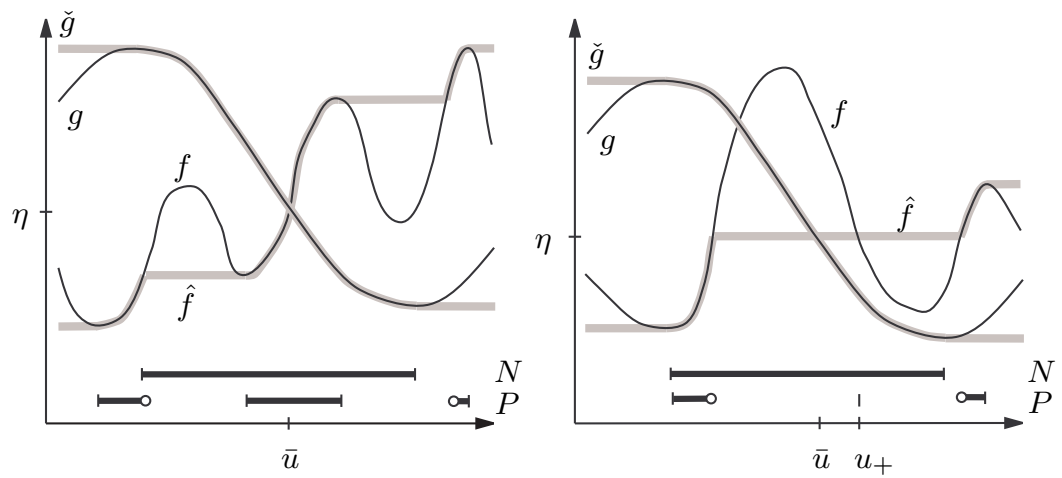

Fig. 2. Left: Case 1, where $\bar{u}=u_{+}=u_{-}$. Right: Case 2a, where $u_{-}=\bar{u}$. The sets $N$ and $P$ are, for clarity, indicated above the $u$-axis.

relation $\left(u_{+}, u_{-}\right)=c\left(u_{+}, u_{-}\right)$holds for almost all $t$, which leads to the following alternative way of formulating the entropy condition.

Condition $\Gamma$ (version 2, $D=0):\left(u_{+}, u_{-}\right)=c\left(u_{+}, u_{-}\right)$for a.e. $t \in(0, T)$.

This fixed-point condition implies that the flux level of the intersection, which we denote by $\eta$, satisfies

$$
\hat{f}\left(\bar{U} ; u_{+}\right)=f\left(u_{+}\right)=\eta=\check{g}\left(\bar{U} ; u_{-}\right)=g\left(u_{-}\right) .
$$

Note also that this version does not require the assumption on piecewise smoothness (but of course the existence of $u_{ \pm}$). Another version written in terms of entropy inequalities is the following.

Condition $\Gamma$ (version 3, $D=0$ ): For a.e. $t \in(0, T)$ and arbitrary $\bar{u} \in \bar{U} \cap$ $\operatorname{ch}\left(u_{-}, u_{+}\right)$,

$$
\begin{aligned}
& \left(u_{+}-\bar{u}\right)(f(v)-\eta) \geq 0 \quad \forall v \in \operatorname{ch}\left(u_{+}, \bar{u}\right) \\
& \left(\bar{u}-u_{-}\right)(g(v)-\eta) \geq 0 \quad \forall v \in \operatorname{ch}\left(u_{-}, \bar{u}\right), \\
& \text { where } \eta=f\left(u_{+}\right)=g\left(u_{-}\right) .
\end{aligned}
$$

Claim 3.2. Versions 2 and 3 of Condition $\Gamma$ are equivalent.

Proof. Given the two numbers $u_{ \pm}$, we can define $\hat{f}, \check{g}, P, N$, the coupling function $c$ and the set of pairs $\bar{U}$. Note also that the Rankine-Hugoniot condition (3.2) is satisfied in both versions. There are three (disjoint) cases that may occur depending on the set $\bar{U}$. (The function values of the coupling function $c$ is written out in these cases in [18].)

Case 1: $\bar{U}=\{\bar{u}\}$ with $\bar{u} \in N \cap P$, see Figure 2 (left). It is clear that the only fixed point in version 2 is $\left(u_{+}, u_{-}\right)=(\bar{u}, \bar{u})$. Then version 3 is trivially satisfied. Conversely, assume that the pair $\left(u_{+}, u_{-}\right)$satisfies version 3 . Assume first that 

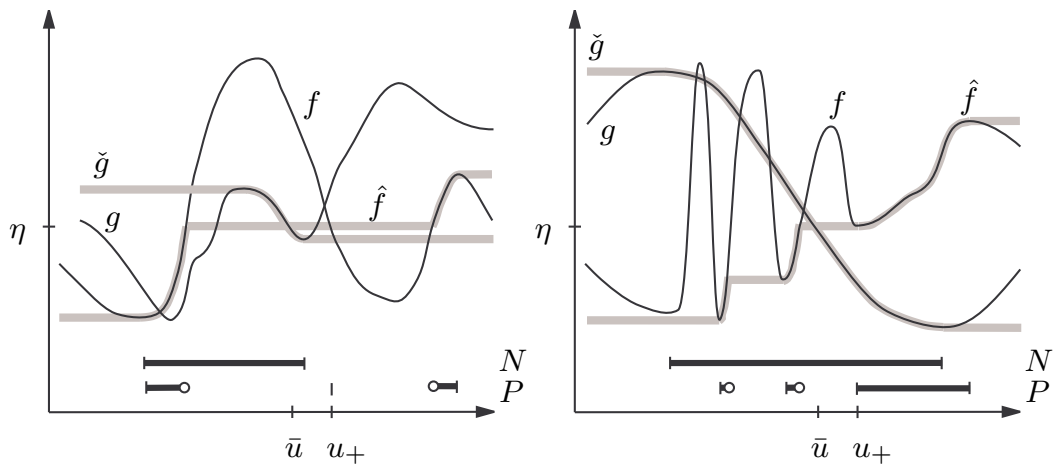

Fig. 3. Two more examples of case $2 \mathrm{a}$. Note that the values of $f$ outside the interval $\left[\bar{u}, u_{+}\right]$are irrelevant, and likewise for $g$ away from $\bar{u}=u_{-}$.

$u_{+}<\bar{u}$. Then (3.5) gives $f(\bar{u}) \leq \eta=f\left(u_{+}\right)$. On the other hand, the facts that $u_{+} \in P$ and the restriction $\left.f\right|_{P}$ is increasing imply that $f\left(u_{+}\right)<f(\bar{u})$, and we have a contradiction. If $u_{+}>\bar{u}$ holds, we arrive in the analogous contradiction with reversed inequalities. Similarly, we get contradictions for the two cases $u_{-} \lessgtr \bar{u}$ (with $g$ instead of $f$ ). The only remaining possibility is $\left(u_{+}, u_{-}\right)=(\bar{u}, \bar{u})$, and we have above concluded that this pair satisfies version 2 .

Case 2a: $\bar{U}=\{\bar{u}\}$ with $\bar{u} \in N \backslash P$. Thus, there is a plateau of $\hat{f}\left(\cdot ; u_{+}\right)$at the flux level of intersection with $\breve{g}\left(\cdot ; u_{-}\right)$, see Figure 2 (right). Version 2 implies (3.4) and specifically $u_{-}=\bar{u}$. Then (3.6) is trivially satisfied. To see that also (3.5) is satisfied we consider first the case when $\bar{u}<u_{+}$, as in Figures 2 (right) and 3. According to the definition of $\hat{f}$ and (3.4), we have

$$
f(v) \geq \min _{w \in\left[v, u_{+}\right]} f(w)=\hat{f}\left(v ; u_{+}\right)=f\left(u_{+}\right)=\eta, \quad \bar{u} \leq v \leq u_{+},
$$

which implies (3.5). The case $u_{+}<\bar{u}$ is analogous:

$$
f(v) \leq \max _{w \in\left[u_{+}, v\right]} f(w)=\hat{f}\left(v ; u_{+}\right)=f\left(u_{+}\right)=\eta, \quad u_{+} \leq v \leq \bar{u},
$$

which also implies (3.5). Conversely, assume that $\left(u_{+}, u_{-}\right)$satisfies version 3. Assume first that $u_{-}<\bar{u}$. Then (3.6) implies that $g(\bar{u}) \geq \eta=g\left(u_{-}\right)$. On the other hand, since the restriction $\left.g\right|_{N}$ is decreasing, we have $g\left(u_{-}\right)>g(\bar{u})$, and we have a contradiction. Analogously, the case $u_{-}>\bar{u}$ yields a contradiction. Hence, $u_{-}=\bar{u}$ holds. There are two possibilities for $u_{+}$. Assume first that $u_{+}>\bar{u}$ holds. Then (3.5) implies that $f(v) \geq \eta=f\left(u_{+}\right)$for all $v \in\left[\bar{u}, u_{+}\right]$. The definition of $\hat{f}$ then implies that $\hat{f}\left(v ; u_{+}\right)=\eta$ for all $v \in\left[\bar{u}, u_{+}\right]$, which in turn implies that $\left(u_{+}, \bar{u}\right)$ is a fixed point of the coupling function $c$. Thus, version 2 is satisfied. The other case $u_{+}<\bar{u}$ yields analogously that version 2 is satisfied.

Case $2 b: \bar{U}=\{\bar{u}\}$ with $\bar{u} \in P \backslash N$. This case is symmetrical to case 2a: substitute $u_{-}$for $u_{+}$and $f$ for $g$. 

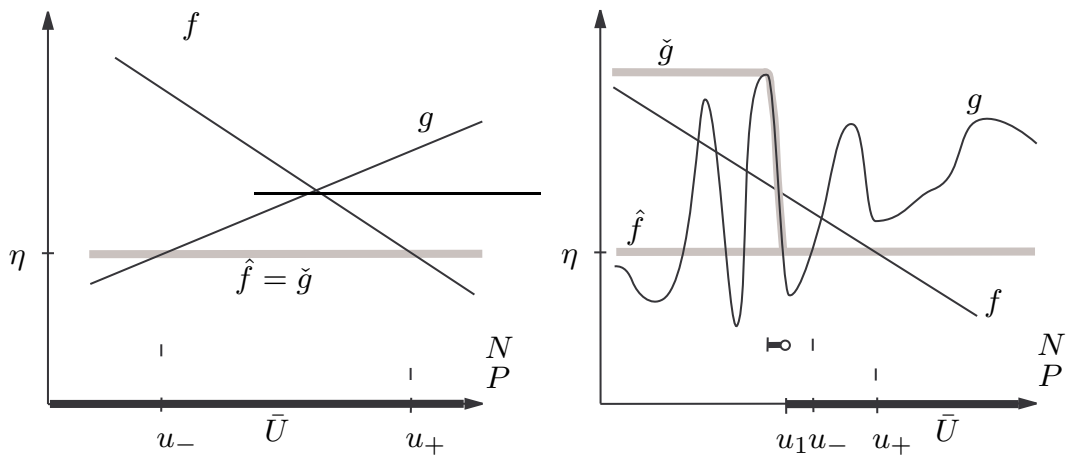

Fig. 4. Examples of case 3 when $\left[u_{-}, u_{+}\right] \subset \bar{U}$. Left: $\bar{U}=\mathbb{R}$. Right: $\bar{U}=\left[u_{1}, \infty\right)$. Note that $\hat{f}\left(u ; u_{+}\right)=\check{g}\left(u ; u_{-}\right)$for $u \geq u_{1}$.
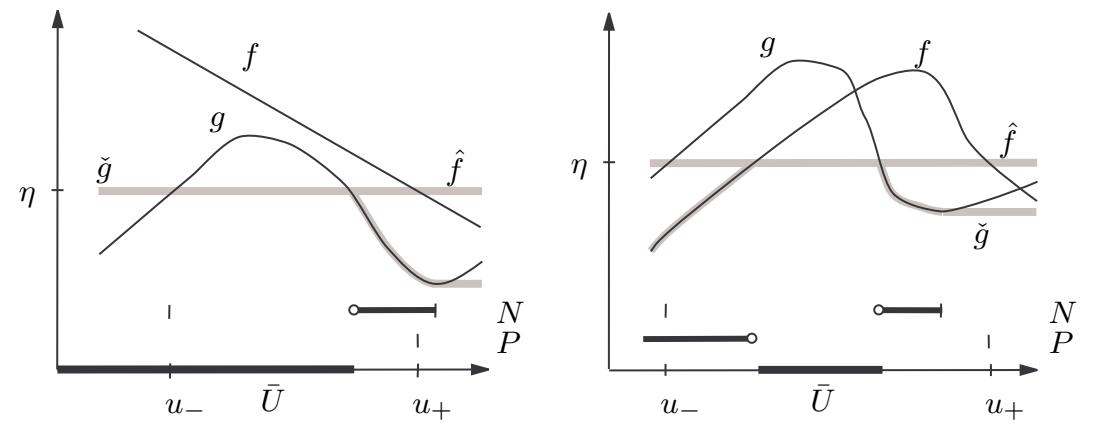

Fig. 5. Examples of case 3 when $\left[u_{-}, u_{+}\right]$is not a subset of $\bar{U}$. Left: $u_{-} \in \bar{U}$ and $u_{+} \notin \bar{U}$. Right: $u_{-} \notin \bar{U}$ and $u_{+} \notin \bar{U}$. Note that the special case when $\bar{U}$ contains only the single point $\bar{u} \notin N \cup P$ is illustrated by the right-hand figure by moving the graph of $g$ to the left (or the graph of $f$ to the right) such that $\bar{U}$ shrinks to a point.

Case 3: Either the set $\bar{U}$ is infinite (an interval of non-zero length) or $\bar{u} \notin$ $N \cup P$. This means that plateaus of both $\hat{f}\left(\cdot ; u_{+}\right)$and $\check{g}\left(\cdot ; u_{-}\right)$lie at the flux level $\eta$ of the intersection. Assume that version 2 holds. If $u_{-}=u_{+}$, then version 3 is trivially satisfied. Consider now the case $u_{-}<u_{+}$. (The case $u_{-}>u_{+}$can be dealt with in a symmetrical way as was done with cases $2 \mathrm{a}$ and $2 \mathrm{~b}$.) Depending on the relative location of the two intervals $\operatorname{ch}\left(u_{-}, u_{+}\right)=\left[u_{-}, u_{+}\right]$and $\bar{U}$ there are different subcases, see Figures 4 and 5 , in which $\left(u_{+}, u_{-}\right)$is a fixed point of the coupling function $c$. In all such cases we can conclude that for every $\bar{u} \in \bar{U} \cap\left[u_{-}, u_{+}\right]$ both (3.7) and the analogous relation

$$
g(v) \geq \min _{w \in\left[u_{-}, v\right]} g(w)=\check{g}\left(v ; u_{-}\right)=g\left(u_{-}\right)=\eta, \quad u_{-} \leq v \leq \bar{u}
$$

hold. Hence, version 3 is satisfied. Conversely, assume that the pair $\left(u_{+}, u_{-}\right)$satisfies 
version 3. In the case $u_{-}=u_{+}$, the pair is clearly a fixed point of the coupling function $c$ in version 2. By symmetry it suffices now to consider the case $u_{-}<u_{+}$. First, we shall conclude that (3.4) holds, i.e., the flux level $\eta=f\left(u_{+}\right)=g\left(u_{-}\right)$equals the level of intersection. The fact that $\hat{f}$ is non-decreasing implies $\eta=f\left(u_{+}\right) \geq$ $\hat{f}\left(\bar{U} ; u_{+}\right)$. Suppose that strict inequality holds, i.e.,

$$
\eta=f\left(u_{+}\right)>\hat{f}\left(\bar{U} ; u_{+}\right) .
$$

This means that $f$ and $\hat{f}\left(\cdot ; u_{+}\right)$have the qualitative relation shown in Figure 1 (left), where the plateau of intersection lies below $f\left(u_{+}\right)$. Let $u_{1}$ denote the right endpoint of that plateau. Hence, $\eta=f\left(u_{+}\right)>f\left(u_{1}\right)=\hat{f}\left(\bar{U} ; u_{+}\right)$. Since $u_{1} \in \bar{U} \cap\left[u_{-}, u_{+}\right]$and $u_{1}<u_{+}$hold, (3.5) in version 3 implies $f\left(u_{1}\right) \geq \eta$. This inequality contradicts (3.8), and therefore (3.4) holds. This means that in the left case of Figure 1, $u_{+}=u_{1}=$ the right endpoint of the plateau involved in the intersection. In any case, there exists a $\bar{u} \in \bar{U} \cap\left[u_{-}, u_{+}\right]$with $u_{-}<\bar{u}<u_{+}$(since $\bar{U}$ is either infinite or $\bar{u} \notin N \cup P$ ). Then (3.5) implies $f(v) \geq \eta=f\left(u_{+}\right)$for $v \in\left[\bar{u}, u_{+}\right]$, and (3.6) implies $g(v) \geq \eta=g\left(u_{-}\right)$ for $v \in\left[u_{-}, \bar{u}\right]$. The definitions of $\hat{f}, \check{g}, P, N$ and $c$ then yield that $\left(u_{+}, u_{-}\right)$is a fixed point of version 2 .

Remark 3.3. In the special case $f=g$, Condition $\Gamma$ implies the standard entropy condition by Oleinik [43], which for a stationary discontinuity can be written

$$
\begin{aligned}
& \left(u_{+}-u_{-}\right)(f(v)-\eta) \geq 0 \quad \forall v \in \operatorname{ch}\left(u_{-}, u_{+}\right), \\
& \text {where } \eta=f\left(u_{-}\right)=f\left(u_{+}\right) .
\end{aligned}
$$

If $u_{-}=u_{+}$, then both (3.9) and Condition $\Gamma$ are satisfied trivially. If $u_{-} \neq u_{+}$, then the intersection of $\hat{f}$ and $\check{f}$ is necessarily of case-3 type. Furthermore, $\bar{U}=$ $\operatorname{ch}\left(u_{-}, u_{+}\right)$holds. Then either of the special choices $\bar{u}=u_{-}$in (3.5) or $\bar{u}=u_{+}$in (3.6) is equivalent to (3.9).

Remark 3.4. Note that the crossing condition, which has been used in $[14,35,36]$ in order to show uniqueness, may not be satisfied when Condition $\Gamma$ is. For example, the number of flux crossings of $f$ and $g$ is irrelevant for Condition $\Gamma$, but the crossing condition allows at most one such.

\section{Entropy condition in the general case}

The general formulation of Condition $\Gamma$, which is (E.2) in Definition 2.2, for the parabolic equation (1.1) is a generalization of Condition $\Gamma$ (version 3 ) in the previous section in two ways. Firstly and obviously, the flux value $\eta$ has to include the diffusion functions. Secondly, it turns out in the proof of uniqueness below that the explicit knowledge of the set $\bar{U}$ is redundant. In the hyperbolic subcase this set is informative when constructing solutions. For the uniqueness it is, however, sufficient to know that there exists a number $\bar{u} \in \operatorname{ch}\left(u_{-}, u_{+}\right)$.

Remark 4.1. As a straightforward generalization of Remark 3.3, Condition $\Gamma$ implies the standard entropy condition for strongly degenerate parabolic equations in 
14 Stefan Diehl

the special case $f=g$ and $A=B$, cf. assumption (E.1) in Definition 2.2, which for a stationary discontinuity can be written as

$$
\begin{aligned}
& \left(u_{+}-u_{-}\right)(f(v)-\eta) \geq 0 \quad \forall v \in \operatorname{ch}\left(u_{-}, u_{+}\right), \\
& \text {where } \eta=f\left(u_{-}\right)-\left(A(u)_{x}\right)_{-}=f\left(u_{+}\right)-\mathcal{A}^{u},
\end{aligned}
$$

see Evje and Karlsen [29].

The following lemma contains some direct consequences of Condition $\Gamma$, which we shall refer to in Section 5.

Lemma 4.2. Condition $\Gamma$ yields the following, where $\bar{u} \in \operatorname{ch}\left(u_{-}, u_{+}\right)$:

$$
\begin{aligned}
& u_{-}<\bar{u} \leq u_{+} \quad \Longrightarrow g(v) \geq \eta \quad \text { for } \quad u_{-} \leq v \leq \bar{u}, \\
& u_{-} \leq \bar{u}<u_{+} \quad \Longrightarrow \quad f(v) \geq \eta \quad \text { for } \quad \bar{u} \leq v \leq u_{+} \text {, } \\
& u_{-}<\bar{u}<u_{+} \Longrightarrow\left\{\begin{array}{lll}
g(v) \geq \eta & \text { for } \quad u_{-} \leq v \leq \bar{u}, \\
f(v) \geq \eta & \text { for } \quad \bar{u} \leq v \leq u_{+},
\end{array}\right. \\
& u_{+}<\bar{u} \leq u_{-} \Longrightarrow f(v) \leq \eta \quad \text { for } \quad u_{+} \leq v \leq \bar{u}, \\
& u_{+} \leq \bar{u}<u_{-} \Longrightarrow g(v) \leq \eta \quad \text { for } \quad \bar{u} \leq v \leq u_{-} \text {, } \\
& u_{+}<\bar{u}<u_{-} \Longrightarrow\left\{\begin{array}{lll}
f(v) \leq \eta & \text { for } & u_{+} \leq v \leq \bar{u} \\
g(v) \leq \eta & \text { for } & \bar{u} \leq v \leq u_{-} .
\end{array}\right.
\end{aligned}
$$

The following properties of the diffusion coefficient on either side of $x=0$ now follow directly. For example, $v=u_{-}$in (4.2) yields $g\left(u_{-}\right) \geq \eta$, which is equivalent to $\mathcal{B}^{u} \geq 0$.

Corollary 4.3. Condition $\Gamma$ implies the following, where $\bar{u} \in \operatorname{ch}\left(u_{-}, u_{+}\right)$:

$$
\begin{array}{lll}
u_{-}<\bar{u} \leq u_{+} & \Longrightarrow & \mathcal{B}^{u} \geq 0, \\
u_{-} \leq \bar{u}<u_{+} & \Longrightarrow & \mathcal{A}^{u} \geq 0, \\
u_{-}<\bar{u}<u_{+} & \Longrightarrow & \left\{\begin{array}{l}
\mathcal{B}^{u} \geq 0, \\
\mathcal{A}^{u} \geq 0,
\end{array}\right. \\
u_{+}<\bar{u} \leq u_{-} & \Longrightarrow & \mathcal{A}^{u} \leq 0, \\
u_{+} \leq \bar{u}<u_{-} & \Longrightarrow & \mathcal{B}^{u} \leq 0, \\
u_{+}<\bar{u}<u_{-} & \Longrightarrow & \left\{\begin{array}{l}
\mathcal{B}^{u} \leq 0, \\
\mathcal{A}^{u} \leq 0 .
\end{array}\right.
\end{array}
$$

For the proof of uniqueness below, Corollary 4.3 is, unfortunately, not sufficiently strong in the cases when $\bar{u}$ is equal to $u_{-}$or $u_{+}$. In those cases, Condition $\Gamma$ implies a sign restriction of only one of $\mathcal{A}^{u}$ and $\mathcal{B}^{u}$. Lemma 5.1 is slightly stronger, but needs the continuity assumption (A.2) for its proof. 
The coupling condition at a spatial discontinuity used in Karlsen et al. [35] and Bürger et al. [15] is the following Kružkov-type inequality. For a.e. $t \in(0, T)$,

$$
\operatorname{sign}\left(u_{+}-v\right)(\eta-f(v))-\operatorname{sign}\left(u_{-}-v\right)(\eta-g(v)) \leq|f(v)-g(v)| \quad \forall v \in \mathbb{R},
$$

where $\eta=f\left(u_{+}\right)-\mathcal{A}^{u}=g\left(u_{-}\right)-\mathcal{B}^{u}$.

Theorem 4.4. Condition $\Gamma$ implies the Kružkov-type entropy condition (4.14).

Proof. For $v \notin \operatorname{ch}\left(u_{-}, u_{+}\right), \operatorname{sign}\left(u_{+}-v\right)=\operatorname{sign}\left(u_{-}-v\right)$ holds and implies that the left-hand side of (4.14) is equal to

$$
\pm(\eta-f(v)) \mp(\eta-g(v))= \pm(g(v)-f(v)) \leq|f(v)-g(v)| .
$$

Consider the case $v \in \operatorname{ch}\left(u_{-}, u_{+}\right)$. If $u_{-}=u_{+}$, then $v=u_{-}=u_{+}$and the left-hand side of (4.14) is zero. Assume now that $u_{-}<u_{+}$(the other case is symmetric). The left-hand side of (4.14) is then equal to

$$
\eta-f(v)+\eta-g(v)=2 \eta-f(v)-g(v) .
$$

One of (4.2), (4.3) and (4.4) holds, and each of these inequalities implies that (4.15) is less than or equal to $g(v)-f(v)$ or $f(v)-g(v)$.

\section{Proof of the uniqueness}

The major part of the proof of Theorem 2.3 follows the one presented by Karlsen et al. [35]. A minor thing is that they consider $A=B$ in (1.1), however, modifications to the case $A \neq B$ are straightforward, cf. [15]. The major modifications are made at the place where the crossing condition and their coupling condition were needed. To handle the relaxed assumption that $u \in L^{\infty}$ instead of $L^{1}$ (which is used in $[15,35])$ a weight function is introduced.

The following three lemmas can be extracted from [35] (where they are proved) and are directly needed here in the modification of the uniqueness proof. They all follow from the continuity assumption (A.2) and the assumption that the diffusion functions $A$ and $B$ are non-decreasing. The first one is Lemma 2.5 in [35]. Note that it is slightly stronger than Corollary 4.3 , since it states that the signs of $\mathcal{A}^{u}$ and $\mathcal{B}^{u}$ are determined by the sign of the jump in $u$. On the other hand, it requires assumption (A.2), which Corollary 4.3 does not.

Lemma 5.1. A weak solution u of (1.1) satisfies for a.e. $t \in(0, T)$

$$
\left(u_{+}-u_{-}\right) \mathcal{A}^{u} \geq 0, \quad\left(u_{+}-u_{-}\right) \mathcal{B}^{u} \geq 0 .
$$

The remaining two lemmas contain some technical details. The next is Lemma 2.2 in [35]. 
Lemma 5.2. Let $u$ and $v$ be two weak solutions. Then the following holds for a.e. $t \in(0, T)$ :

$$
\begin{aligned}
& \left(|A(u)-A(v)|_{x}\right)_{+}= \begin{cases}\operatorname{sign}\left(u_{+}-v_{+}\right)\left(\mathcal{A}^{u}-\mathcal{A}^{v}\right), & \text { if } A\left(u_{+}\right) \neq A\left(v_{+}\right), \\
\left|\mathcal{A}^{u}-\mathcal{A}^{v}\right|, & \text { if } A\left(u_{+}\right)=A\left(v_{+}\right),\end{cases} \\
& \left(|B(u)-B(v)|_{x}\right)_{-}= \begin{cases}\operatorname{sign}\left(u_{-}-v_{-}\right)\left(\mathcal{B}^{u}-\mathcal{B}^{v}\right), & \text { if } B\left(u_{-}\right) \neq B\left(v_{-}\right), \\
-\left|\mathcal{B}^{u}-\mathcal{B}^{v}\right|, & \text { if } B\left(u_{-}\right)=B\left(v_{-}\right) .\end{cases}
\end{aligned}
$$

The whole Lemma 2.9 in [35] is not needed in the uniqueness proof below, but only the following main parts.

Lemma 5.3. Let $u$ and $v$ be two weak solutions, which satisfy $u_{-}>v_{-}$and $u_{+}<$ $v_{+}$for a fixed $t \in(0, T)$ (such that all traces exist). Then the following holds: If $u_{+}<u_{-}$, then

$$
\begin{aligned}
& v_{-} \in\left(u_{+}, u_{-}\right) \quad \Longrightarrow \quad \mathcal{B}^{v}=0, \\
& v_{+} \in\left(u_{+}, u_{-}\right) \quad \Longrightarrow \quad \mathcal{A}^{v}=0 .
\end{aligned}
$$

If $v_{-}<v_{+}$, then

$$
\begin{aligned}
& u_{-} \in\left(v_{-}, v_{+}\right) \quad \Longrightarrow \quad \mathcal{B}^{u}=0, \\
& u_{+} \in\left(v_{-}, v_{+}\right) \quad \Longrightarrow \quad \mathcal{A}^{u}=0 .
\end{aligned}
$$

Proof of Theorem 2.3. The proof of Theorem 2.1 in Karlsen et al. [35] can be followed with the following modifications. Straightforward modifications in the notation are needed to cover the case $A \neq B$ instead of $A=B$ (which is also noticed by these authors in [15]). Consider condition (E.1) in Definition 2.2. Since $A$ is non-decreasing, we can rewrite

$$
\operatorname{sign}(u-c) A(u)_{x}=\operatorname{sign}(A(u)-A(c))(A(u)-A(c))_{x}=|A(u)-A(c)|_{x}
$$

and since $A(u(\cdot, t))$ is continuous, we can move the partial derivative with respect to $x$ to the test function. The same can be done for the term with $B$ and hence we have

$$
\begin{gathered}
\iint_{\Pi_{T}}\left(|u-c| \varphi_{t}+\operatorname{sign}(u-c)(F(u, x)-F(c, x)) \varphi_{x}\right. \\
\left.+|D(u, x)-D(c, x)| \varphi_{x x}\right) d x d t \geq 0
\end{gathered}
$$

for all $0 \leq \varphi \in C_{0}^{\infty}\left(\Pi_{T} \backslash\{(0, t): 0<t<T\}\right)$ and any constant $c \in \mathbb{R}$. Starting from this inequality and the weak formulation (A.3), the 'doubling of variables' argument in [35, Appendix A] yields the following inequality for two weak entropy solutions $u$ and $v$ :

$$
\begin{gathered}
\iint_{\Pi_{T}}\left(|u-v| \varphi_{t}+\operatorname{sign}(u-v)(F(u, x)-F(v, x)) \varphi_{x}\right. \\
\left.+|D(u, x)-D(v, x)| \varphi_{x x}\right) d x d t \geq 0
\end{gathered}
$$


for all $0 \leq \varphi \in C_{0}^{\infty}\left(\Pi_{T} \backslash\{(0, t): 0<t<T\}\right)$. Via a limiting argument this inequality is, in [35], extended to the larger class of test functions $0 \leq \varphi \in C_{0}^{\infty}\left(\Pi_{T}\right)$ provided that the following inequality

$$
\begin{gathered}
S:=\operatorname{sign}\left(u_{+}-v_{+}\right)\left(f\left(u_{+}\right)-f\left(v_{+}\right)\right)-\left(|A(u)-A(v)|_{x}\right)_{+} \\
-\operatorname{sign}\left(u_{-}-v_{-}\right)\left(g\left(u_{-}\right)-g\left(v_{-}\right)\right)+\left(|B(u)-B(v)|_{x}\right)_{-} \leq 0
\end{gathered}
$$

holds at an arbitrary time point when the traces exist. The inequality (5.2) corresponds to [35, Equation (2.27)] (see also [15, Equation (4.38)]) and it was proved by considering 7 cases depending on the relative order of the numbers $u_{-}$and $v_{-}$, and $u_{+}$and $v_{+}$. It is only case 6 (and thereby also automatically the symmetrical case 7 ) that requires a modification, since this required the crossing condition and their entropy coupling condition. In case $6, \underline{u_{-}>v_{-} \text {and } u_{+}<v_{+}}$hold. Lemma 5.2 then implies that

$$
\begin{gathered}
S=-\left(f\left(u_{+}\right)-f\left(v_{+}\right)\right)+\left(\mathcal{A}^{u}-\mathcal{A}^{v}\right)-\left(g\left(u_{-}\right)-g\left(v_{-}\right)\right)+\left(\mathcal{B}^{u}-\mathcal{B}^{v}\right) \\
=2\left(\eta^{v}-\eta^{u}\right),
\end{gathered}
$$

where $\eta^{v}:=f\left(v_{+}\right)-\mathcal{A}^{v}=g\left(v_{-}\right)-\mathcal{B}^{v}$ and analogously for $\eta^{u}$. Now we consider several subcases, which in turn is divided into further subcases, depending on the order of the six numbers $u_{ \pm}, v_{ \pm}, \bar{u}$ and $\bar{v}$. To make it easier for the reader, the inequalities that distinguish each subcase are underlined.

I. $\underline{u_{+}<v_{-}}:$We have

$$
\begin{aligned}
& u_{+}<v_{-}<u_{-}, \\
& u_{+}<v_{+} .
\end{aligned}
$$

Equation (5.3) implies, by Lemma 5.3, that $\mathcal{B}^{v}=0$. We now consider different possible placements of $v_{+}$:

a. $u_{+}<\underline{v_{+}}<v_{-}<u_{-}$: Lemma 5.3 then gives that also $\mathcal{A}^{v}=0$ holds.

We now consider different possible placements of $\bar{u} \in\left[u_{+}, u_{-}\right]$:

i. $u_{+} \leq \bar{u} \leq v_{+}<v_{-}<u_{-}$: Then (4.6) implies

$$
g\left(v_{-}\right) \leq \eta^{u} \quad \Longrightarrow \quad S \leq 2\left(\eta^{v}-g\left(v_{-}\right)\right)=-2 \mathcal{B}^{v}=0 .
$$

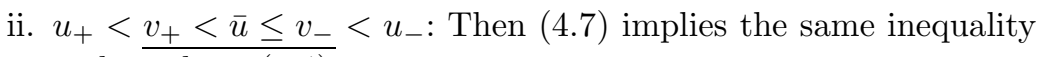
and result as (5.4).

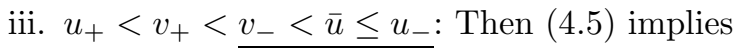

$$
f\left(v_{+}\right) \leq \eta^{u} \quad \Longrightarrow \quad S \leq 2\left(\eta^{v}-f\left(v_{+}\right)\right)=-2 \mathcal{A}^{v}=0 .
$$

b. $u_{+}<\underline{v_{+}=v_{-}}<u_{-}$: Necessarily, $\bar{v}=v_{+}=v_{-}$holds and $\mathcal{A}^{v}=0$ by Lemma 5.3. Consider different possible placements of $\bar{u}$ :

i. $u_{+} \leq \bar{u} \leq \bar{v}<u_{-}$: Then (4.6) implies $g(\bar{v}) \leq \eta^{u} \quad \Longrightarrow \quad S \leq 2\left(\eta^{v}-g(\bar{v})\right)=2\left(\eta^{v}-g\left(v_{-}\right)\right)=-2 \mathcal{B}^{v}=0$. 
ii. $u_{+}<\bar{v}<\bar{u} \leq u_{-}$: Then (4.5) implies

$$
f(\bar{v}) \leq \eta^{u} \quad \Longrightarrow \quad S \leq 2\left(\eta^{v}-f(\bar{v})\right)=2\left(\eta^{v}-g\left(v_{+}\right)\right)=-2 \mathcal{A}^{v}=0 .
$$

c. $u_{+}<v_{-}<v_{+}<u_{-}$: Lemma 5.3 implies that $\mathcal{A}^{v}=0$. Consider different possible placements of $\bar{u}$ :

i. $u_{+} \leq \bar{u} \leq v_{-}<v_{+}<u_{-}$: Then (4.6) implies (5.4).

ii. $\overline{u_{+}<v_{-}<\bar{u}}<v_{+}<u_{-}$: Then (4.7) implies

$$
\begin{aligned}
& f(\bar{u}) \leq \eta^{u}, \\
& g(\bar{u}) \leq \eta^{u} .
\end{aligned}
$$

Consider different placements of $\bar{v} \in\left[v_{-}, v_{+}\right]$:

$\alpha . v_{-} \leq \underline{\bar{v}<\bar{u}} \leq v_{+}$: Then (4.3) (in the variable $v$ ) implies $f(\bar{u}) \geq \eta^{v}$. This inequality together with (5.6) gives

$$
S \leq 2(f(\bar{u})-f(\bar{u}))=0 .
$$

B. $v_{-}<\underline{\bar{u} \leq \bar{v}} \leq v_{+}$: Analogously to case $\alpha,(4.2)$ implies $g(\bar{u}) \geq$ $\eta^{v}$. Together with (5.7) we conclude that $S \leq 0$.

iii. $u_{+}<v_{-}<\underline{v_{+} \leq \bar{u} \leq u_{-}}$: Then (4.5) implies (5.5).

d. $u_{+}<v_{-}<\underline{u_{-} \leq v_{+}}$: Consider different possible placements of $\bar{u}$ :

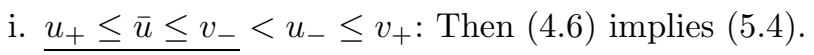

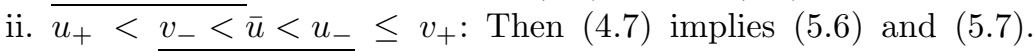
Consider different placements of $\bar{v} \in\left[v_{-}, v_{+}\right]$:

$\alpha . v_{-} \leq \underline{\bar{v}<\bar{u}}<v_{+}$: Then (4.3) (in the variable $v$ ) implies $f(\bar{u}) \geq \eta^{v}$. This inequality together with (5.6) implies $S \leq 0$.

$\beta . v_{-}<\bar{u} \leq \bar{v} \leq v_{+}$: Analogously to case $\alpha$, (4.2) implies $g(\bar{u}) \geq$ $\eta^{v}$. Together with (5.7) we can conclude that $S \leq 0$ holds.

iii. $u_{+}<v_{-}<\bar{u}=u_{-} \leq v_{+}$: Then (4.5) implies (5.6). Consider different placements of $\bar{v} \in\left[v_{-}, v_{+}\right]$:

$\alpha . v_{-} \leq \underline{\bar{v}<\bar{u}}=u_{-} \leq v_{+}$: Then (4.3) (in the variable $v$ ) implies $f(\bar{u}) \geq \eta^{v}$. This inequality together with (5.6) implies $S \leq 0$.

$\beta . v_{-}<\bar{u}=u_{-} \leq \bar{v} \leq v_{+}$: Inequality (4.2) (in the variable $v$ ) implies

$$
g\left(u_{-}\right) \geq \eta^{v} \quad \Longrightarrow \quad S \leq 2\left(g\left(u_{-}\right)-\eta^{u}\right)=2 \mathcal{B}^{u} \leq 0,
$$

where the latter inequality follows from Lemma 5.1.

II. $\underline{u_{+}=v_{-}}$: We have

$$
\begin{aligned}
& u_{+}=v_{-}<u_{-}, \\
& u_{+}<v_{+} .
\end{aligned}
$$

We now consider the possible placements of $v_{+}$: 
a. $u_{+}=v_{-}<v_{+}<u_{-}$: Then Lemma 5.3 implies $\mathcal{A}^{v}=0$. Consider different placements of $\bar{u}$ :

i. $u_{+}=v_{-}=\bar{u}<v_{+}<u_{-}$: Inequality (4.6) implies $g(\bar{u}) \leq \eta^{u} \quad \Longrightarrow \quad S \leq 2\left(\eta^{v}-g(\bar{u})\right)=2\left(\eta^{v}-g\left(v_{-}\right)\right)=-2 \mathcal{B}^{v} \leq 0$, where the latter inequality follows from Lemma 5.1.

ii. $u_{+}=v_{-}<\bar{u}<v_{+}<u_{-}$: The same arguments as in case Icii can be applied.

iii. $u_{+}=v_{-}<v_{+} \leq \bar{u} \leq u_{-}$: The same arguments as in case Iciii can be applied.

b. $u_{+}=v_{-}<\underline{u_{-} \leq v_{+}}$: Consider different placements of $\bar{u}$ :

i. $u_{+}=v_{-}=\bar{u}<u_{-} \leq v_{+}$: The same arguments as in case IIai can be applied.

ii. $u_{+}=v_{-}<\bar{u}<u_{-} \leq v_{+}$: The same arguments as in case Idii can be applied.

iii. $u_{+}=v_{-}<\bar{u}=u_{-} \leq v_{+}$: The same arguments as in case Idiii can be applied.

III. $u_{+}>v_{-}$: We have

$$
\begin{aligned}
& v_{-}<u_{+}<v_{+}, \\
& v_{-}<u_{-} .
\end{aligned}
$$

By interchanging $u$ and $v$, and minus and plus signs, we see that this case is completely analogous to I.

Hence, (5.2) holds and thereby (5.1) for test functions $0 \leq \varphi \in C_{0}^{\infty}\left(\Pi_{T}\right)$. A modification at the end of the proof in [35] is now done to deal with the fact that $u \in L^{\infty}\left(\Pi_{T}\right)$ instead of $L^{1}\left(\Pi_{T}\right)$. For a given $s \in(0, T)$, let $s_{0}, \tau$ and $\kappa$ satisfy $0<s_{0}<s_{0}+\tau<s<s+\kappa<T$ and define

$$
\beta_{\tau, \kappa}(t):= \begin{cases}0, & 0 \leq t \leq s_{0}, \\ \frac{1}{\tau}\left(t-s_{0}\right), & s_{0} \leq t \leq s_{0}+\tau, \\ 1, & s_{0}+\tau \leq t \leq s, \\ -\frac{1}{\kappa}(t-s-\kappa), & s \leq t \leq s+\kappa, \\ 0, & s+\kappa \leq t \leq T .\end{cases}
$$

For a real number $a$, set

$$
\mu_{a}(x):=\frac{1}{1+(x-a)^{2}}, \quad x \in \mathbb{R} .
$$

Then $0<\mu_{a} \leq 1$ holds and one can easily verify that $\left|\mu_{a}^{\prime}\right| \leq C_{1} \mu_{a}$ and $\left|\mu_{a}^{\prime \prime}\right| \leq C_{2} \mu_{a}$ for some constants $C_{1}$ and $C_{2}$, which are independent of $a$ (since the effect of $a$ is 
merely a translation). For $N>0$, we define the weight function

$$
w_{N}(x):= \begin{cases}\mu_{-N}(x), & x \leq-N, \\ 1, & |x| \leq N \\ \mu_{N}(x), & x \geq N,\end{cases}
$$

and conclude that $w_{N} \in C^{1}(\mathbb{R}), w_{N}^{\prime}(x)=w_{N}^{\prime \prime}(x)=0$ for $|x|<N,\left|w_{N}^{\prime}\right| \leq C_{1} w_{N}$ and $\left|w_{N}^{\prime \prime}\right| \leq C_{2} w_{N}$ with the same constants as above. Let $h \in C^{\infty}(\mathbb{R})$ take values in $[0,1]$ such that

$$
h(x):= \begin{cases}0, & x \leq 0 \\ 1, & x \geq 1\end{cases}
$$

For $r>N$ set

$$
\alpha_{r}(x):=h(x+r+1)-h(x-r), \quad x \in \mathbb{R} .
$$

Then $\operatorname{supp} \alpha_{r} \subseteq[-r-1, r+1], 0 \leq \alpha_{r} \leq 1,\left|\alpha_{r}^{\prime}\right| \leq\left\|h^{\prime}\right\|_{L^{\infty}(\mathbb{R})}$ and $\left|\alpha_{r}^{\prime \prime}\right| \leq\left\|h^{\prime \prime}\right\|_{L^{\infty}(\mathbb{R})}$. To obtain compact support, define

$$
w_{N, r}:=w_{N} \alpha_{r} .
$$

Then we can conclude that $0 \leq w_{N, r} \leq 1, w_{N, r} \nearrow w_{N}$ as $r \rightarrow \infty, w_{N, r}^{\prime}(x)=$ $w_{N, r}^{\prime \prime}(x)=0$ for $|x|<N$ and

$$
\begin{aligned}
& \left|w_{N, r}^{\prime}\right| \leq\left(C_{1}+\left\|h^{\prime}\right\|_{L^{\infty}(\mathbb{R})}\right) w_{N}=: C_{3} w_{N}, \\
& \left|w_{N, r}^{\prime \prime}\right| \leq\left(C_{2}+2 C_{1}\left\|h^{\prime}\right\|_{L^{\infty}(\mathbb{R})}+\left\|h^{\prime \prime}\right\|_{L^{\infty}(\mathbb{R})}\right) w_{N}=: C_{4} w_{N},
\end{aligned}
$$

where we note that $C_{3}$ and $C_{4}$ neither depend on $r$ nor $N$. Via a standard regularization argument, $\varphi(x, t)=w_{N, r}(x) \beta_{\tau, \kappa}(t)$ can be used as a test function in (5.1), which then yields

$$
\begin{gathered}
\frac{1}{\kappa} \int_{s}^{s+\kappa} \int_{\mathbb{R}}|u-v| w_{N, r} d x d t \leq \frac{1}{\tau} \int_{s_{0}}^{s_{0}+\tau} \int_{\mathbb{R}}|u-v| w_{N, r} d x d t \\
\quad+C_{3} \int_{s_{0}}^{s+\kappa} \int_{|x|>N}|F(u, x)-F(v, x)| w_{N}(x) d x d t \\
+C_{4} \int_{s_{0}}^{s+\kappa} \int_{|x|>N}|D(u, x)-D(v, x)| w_{N}(x) d x d t .
\end{gathered}
$$

Since $f, g, A$ and $B$ are Lipschitz, the last two terms in (5.9) can be estimated by

$$
C \int_{s_{0}}^{s+\kappa} \int_{|x|>N}|u-v| w_{N} d x d t \leq C \int_{s_{0}}^{s+\kappa} \int_{\mathbb{R}}|u-v| w_{N} d x d t,
$$


for some constant $C$, which is independent of $r$ and $N$. Let $s_{0} \searrow 0$ and use the triangle inequality on the first term on the right-hand side of (5.9) to obtain

$$
\begin{gathered}
\frac{1}{\kappa} \int_{s}^{s+\kappa} \int_{\mathbb{R}}|u-v| w_{N, r} d x d t \leq \int_{\mathbb{R}}\left|u_{0}-v_{0}\right| w_{N, r} d x+\frac{1}{\tau} \int_{0}^{\tau} \int_{-r-1}^{r+1}\left|u-u_{0}\right| d x d t \\
+\frac{1}{\tau} \int_{0}^{\tau} \int_{-r-1}^{r+1}\left|v-v_{0}\right| d x d t+C \int_{0}^{s+\kappa} \int_{\mathbb{R}}|u-v| w_{N} d x d t .
\end{gathered}
$$

By assumption (A.4) in Definition 2.1, the second and third terms on the right-hand side tend to zero as $\tau \searrow 0$. Then we can let $r \rightarrow \infty$ in (5.10) so that $w_{N, r}$ is replaced by $w_{N}$. Defining

$$
\mathcal{E}(t):=\int_{\mathbb{R}}|u-v| w_{N} d x,
$$

(5.10) thus yields

$$
\frac{1}{\kappa} \int_{s}^{s+\kappa} \mathcal{E}(t) d t \leq \mathcal{E}(0)+C \int_{0}^{s+\kappa} \mathcal{E}(t) d t .
$$

By Fubini's theorem, $\mathcal{E} \in L^{1}(0, T)$. For $s$ being a Lebesgue point of $\mathcal{E}$, we can let $\kappa \searrow 0$ and get

$$
\mathcal{E}(s) \leq \mathcal{E}(0)+C \int_{0}^{s} \mathcal{E}(t) d t .
$$

Since the set of Lebesgue points of $\mathcal{E}$ has full measure, Grönwall's lemma gives finally

$$
\mathcal{E}(s) \leq e^{C s} \mathcal{E}(0), \quad \text { a.e. } s \in(0, T) .
$$

\section{Construction of piecewise smooth stationary solutions}

In order to provide examples of jumps at $x=0$, we consider here time-independent piecewise smooth solutions $u=u(x)$ of (1.1). The simple geometric interpretation of Condition $\Gamma$ will thereby be enlightened. The constructed solutions below naturally contain examples of the different types of hyperbolic waves of Section 3 (cases 1-3), i.e., from undercompressive to overcompressive waves.

For stationary solutions, equation (1.1) can be reformulated in the following way in the weak sense, having the Rankine-Hugoniot condition at every discontinuity of $u(x)$ in mind,

$$
\begin{aligned}
&\left(F(u, x)-D(u, x)^{\prime}\right)^{\prime}=0, \quad x \in \mathbb{R} \\
& \Longleftrightarrow \quad \eta=F(u, x)-D(u, x)^{\prime}, \quad x \in \mathbb{R} \\
& \Longleftrightarrow \quad \begin{cases}\eta=g(u)-B(u)^{\prime}, & x<0, \\
\eta=f\left(u_{+}\right)-\mathcal{A}^{u}=g\left(u_{-}\right)-\mathcal{B}^{u}, & x=0, \\
\eta=f(u)-A(u)^{\prime}, & x>0,\end{cases}
\end{aligned}
$$


for some constant $\eta \in \mathbb{R}$. These equations include possible jumps away from $x=0$. For example, a jump between $u\left(x_{0}-0\right)$ and $u\left(x_{0}+0\right)$ at $x_{0}<0$ satisfies

$$
\left.\left(g(u)-B(u)^{\prime}\right)\right|_{x=x_{0}-0}=\eta=\left.\left(g(u)-B(u)^{\prime}\right)\right|_{x=x_{0}+0} .
$$

When examining possible stationary solutions it is thus natural to start from a constant value of $\eta$, which can be interpreted as the physical constant flux for all $x$. If there is no diffusion, $\eta$ equals the convective flux. When there is diffusion present, the diffusion term $B(u)^{\prime}$ is the difference between the convective flux and $\eta$.

In intervals of $x \in \mathbb{R}$ where the equation is hyperbolic ('hyperbolic intervals') it follows that the solution is piecewise constant. In the 'parabolic intervals', $A^{\prime}>0$ or $B^{\prime}>0$ holds (positive diffusion), wherefore the solution obeys the ordinary differential equation

$$
u^{\prime}=\frac{g(u)-\eta}{B^{\prime}(u)} \text { in parabolic intervals in } x<0
$$

and the corresponding one in $x>0$ containing $f$ and $A$. Hence, we have the following geometrically appealing equivalence:

$$
g(u) \lesseqgtr \eta \quad \Longleftrightarrow \quad u^{\prime} \lesseqgtr 0 \quad \text { in parabolic intervals in } x<0
$$

and analogously for $x>0$ and $f$. Hence, the solution is increasing, if and only if the graph of $g$ lies above the flux value $\eta$. Note the similarity with Condition $\Gamma$, which says that a jump at $x=0$ with $u_{-}<u_{+}$is correct if and only if there exists a number $\bar{u} \in\left[u_{-}, u_{+}\right]$such that the graphs of $g$ and $f$ lie above the flux value $\eta$ in the intervals $\left(u_{-}, \bar{u}\right)$ and $\left(\bar{u}, u_{+}\right)$, respectively. The same interpretation holds for jumps away from $x=0$, however, with $\bar{u}=u_{-}$or $\bar{u}=u_{+}$, see (4.1). This property also holds for the purely hyperbolic case, which enlightens the connection between Condition $\Gamma$ and the vanishing viscosity method (the diffusion tends to zero).

Because of the continuity assumption (A.2) in Definition 2.1, no discontinuity of $u$ is possible in a parabolic interval, since the diffusion functions are strictly increasing there. Equivalently, a jump discontinuity is possible only if the left and right limits lie in a closed interval where the diffusion function is constant. At $x=0$, both $A$ and $B$ have to be constant, but not necessarily the same constant. We write this important fact in a lemma, cf. Evje and Karlsen [29].

Lemma 6.1. Given a jump discontinuity at a point $x=x_{0}$, i.e. $u\left(x_{0}-\right) \neq u\left(x_{0}+\right)$. If $x_{0} \geq 0$, then $u\left(x_{0}-\right), u\left(x_{0}+\right) \in\left[\alpha_{1}, \alpha_{2}\right]$ where $A^{\prime}(v)=0$ for $v \in\left(\alpha_{1}, \alpha_{2}\right)$. If $x_{0} \leq$ 0 , then $u\left(x_{0}-\right), u\left(x_{0}+\right) \in\left[\beta_{1}, \beta_{2}\right]$ where $B^{\prime}(v)=0$ for $v \in\left(\beta_{1}, \beta_{2}\right)$. In particular, $u_{-}, u_{+} \in\left[\max \left(\alpha_{1}, \beta_{1}\right), \min \left(\alpha_{2}, \beta_{2}\right)\right]$.

Note that $A\left(u_{+}\right) \neq B\left(u_{-}\right)$may hold. For sufficiently regular solutions, the monotonicity properties of Lemma 5.1 can be extended. Note that the entropy condition is not needed in the following theorem.

Theorem 6.2. A time-independent piecewise smooth and piecewise monotone weak solution of (1.1) which has a jump discontinuity at $x=0$, i.e. $u_{-} \neq u_{+}$, is monotone in a neighbourhood of $x=0$. 

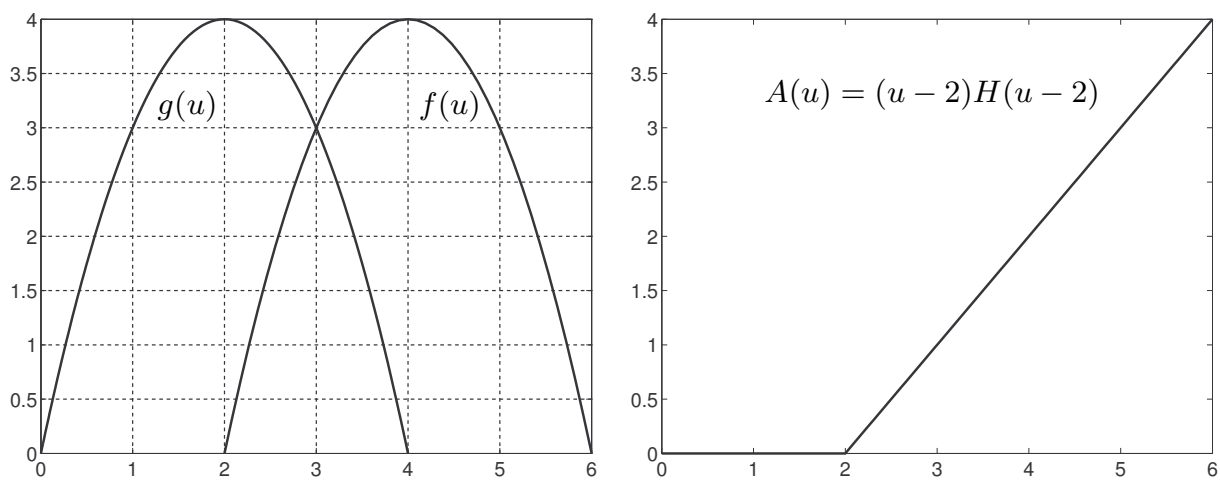

Fig. 6. The flux functions $g$ and $f$ and the diffusion function $A$ in the case $u_{\alpha}=2$.

Proof. Consider a sufficiently small neighbourhood of $x=0$ within which the only jump is at $x=0$. If the solution is constant on both sides of $x=0$, the statement is true. By Lemma 6.1, $u_{-}, u_{+} \in\left[\beta_{1}, \beta_{2}\right]$ where $B$ is constant. Assume first that the solution is increasing on the left-hand side. Then it is parabolic there and satisfies (6.1) with $B^{\prime}(u)>0$. Since $u$ is increasing, $u_{-}=\beta_{1}$ has to hold. Thus, $\beta_{1}=u_{-}<u_{+} \leq \beta_{2}$ holds. If $u$ is constant on the right side of $x=0$, the theorem is true. Otherwise, $u$ is either increasing or decreasing on the right-hand side of $x=0$. Hence, it is parabolic there and $u_{+}$is an endpoint of an interval $\left[\alpha_{1}, \alpha_{2}\right]$ where $A$ is constant. Lemma 6.1 gives that also $u_{-} \in\left[\alpha_{1}, \alpha_{2}\right]$. Since $u_{-}<u_{+}$, the only possibility is that $u_{+}=\alpha_{2}$. Consequently, in a right neighbourhood of $x=0$, the solution satisfies $u>\alpha_{2}=u_{+}$, which means that it is increasing. Finally, if the solution is decreasing on the left, the analogous arguments imply that the solution is decreasing in a whole neighbourhood of $x=0$.

For the stationary solutions constructed below, we use the notation

$$
u(x)= \begin{cases}u_{1}(x), & x<0, \\ u_{\mathrm{r}}(x), & x>0 .\end{cases}
$$

Then, $u_{1}(0-)=u_{-}$and $u_{\mathrm{r}}(0+)=u_{+}$hold.

\subsection{A family of examples}

We choose the following flux and diffusion functions:

$$
\begin{array}{ll}
f(u)=(u-2)(6-u), & A(u)=\left(u-u_{\alpha}\right) H\left(u-u_{\alpha}\right), \\
g(u)=u(4-u), & B(u)=0,
\end{array}
$$

where $H$ is the Heaviside step function and $u_{\alpha}$ is a constant, see Figure 6 . We can directly conclude the following: 
- Since $B=0$, the restriction $u_{1}$ of the solution is piecewise constant.

- By Lemma 6.1, the restriction $u_{r}$ of the solution is parabolic in an open $x$-interval if and only if $u_{\mathrm{r}}>u_{\alpha}$ holds there.

- By Lemma 6.1, $u_{-} \neq u_{+}$implies $\max \left(u_{+}, u_{-}\right) \leq u_{\alpha}$. The same is true for any discontinuity in $x>0$. Hence, the only possibility for a discontinuity between a hyperbolic and a parabolic region is that the limit value on the parabolic side is $u_{\alpha}$.

- If there is a discontinuity at $x=x_{1} \geq 0$ and the solution is parabolic in $x>x_{1}$, then, since $A^{\prime}(u)=H\left(u-u_{\alpha}\right)=1$, it satisfies the one-sided boundary-value problem

$$
\begin{aligned}
& u_{\text {par }}^{\prime}=f\left(u_{\text {par }}\right)-\eta, \quad x>x_{1}, \\
& u_{\text {par }}\left(x_{1}\right)=u_{\alpha} .
\end{aligned}
$$

Hence, it satisfies, in a right neighbourhood of $x=x_{1}$,

$$
f\left(u_{\text {par }}\right)>\eta \text {. }
$$

Depending on the flux value $\eta$ and the constant $u_{\alpha}$ we shall see that either there exists no stationary solution, there is a unique one or there may be a whole family of such. From the graph of $g$ we can directly conclude that there exists no solution for $\eta>4$. We start with the flux value at which the graphs intersect.

\subsubsection{The constant flux is $\eta=3$}

Consider first $x<0$. The equation $\eta=g(u)$ has the two solutions $u=1$ and $u=3$. By Oleinik's entropy inequality (3.9), the only possible jump at a point $x_{0}<0$ is from 1 to 3 in the $x$-direction. The solution on the left-hand side is thus one of the following:

$$
\begin{aligned}
& u_{1}(x) \equiv 1, \\
& u_{1}(x)= \begin{cases}1, & x<x_{0}, \\
3, & x_{0}<x<0,\end{cases} \\
& u_{1}(x) \equiv 3,
\end{aligned}
$$

for some constant $x_{0}<0$. From (6.4) and the graph of $f$ we can conclude that the only possibility for a parabolic solution in a right neighbourhood of $x=x_{1} \geq 0$ is if $3<u_{\alpha}<5$. The solution of (6.3) for $3<u_{\alpha}<5$ is

$$
u_{\mathrm{par}}(x)=\frac{5 e^{2\left(x-x_{1}\right)}\left(u_{\alpha}-3\right)+3\left(5-u_{\alpha}\right)}{e^{2\left(x-x_{1}\right)}\left(u_{\alpha}-3\right)+5-u_{\alpha}}, \quad x>x_{1} .
$$

Note that it increases to 5 as $x \rightarrow \infty$. For other values of $u_{\alpha}$, the only possibility is a hyperbolic piecewise constant solution. The equation $\eta=f(u)$ yields the two solutions $u=3$ and $u=5$. By the entropy condition, the only possibility for a jump between these values is from 3 to 5 in the direction of the $x$-axis. Hence, we can conclude the following. 

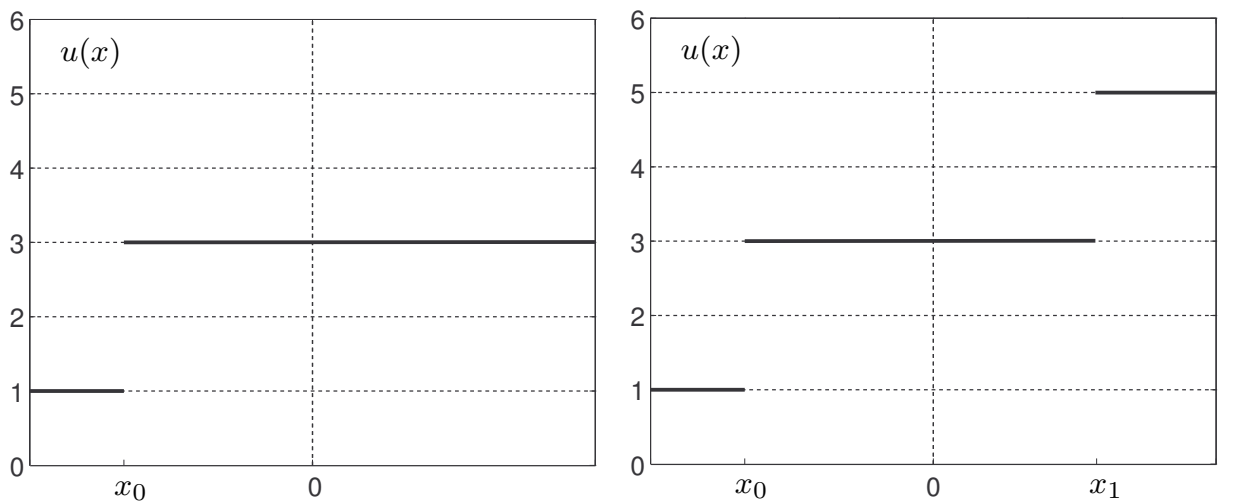

Fig. 7. Stationary solutions when $\eta=3$. Left: The case $u_{\alpha}<3$, where the discontinuity is located at $x_{0}<0$. Right: The case $u_{\alpha} \geq 5$, where $x_{0} \leq 0$ and $x_{1} \geq 0$.

- $\underline{u_{\alpha}<3}$ : A parabolic solution on the right-hand side of $x=0$ is not possible, since this required $3<u_{\alpha}<5$.

$* \underline{u_{-}=1}$ : Since $\max \left(u_{-}, u_{+}\right) \leq u_{\alpha}<3$ is required, a hyperbolic solution on the right of $x=0$ with $u_{+}=3$ or $u_{+}=5$ is also ruled out. Hence, no stationary solution exists.

$* \underline{u_{-}=3}$ : The solution on the left-hand side is either (6.6) or (6.7) and

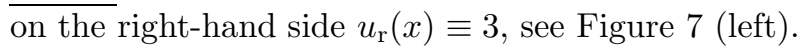

- $3 \leq u_{\alpha}<5$ : The solution on the left-hand side is either (6.5), (6.6) or (6.7). With $u_{\text {par }}$ defined by (6.8), there is a parameter family of solutions on the right-hand side:

$$
u_{\mathrm{r}}(x)= \begin{cases}3, & 0<x<x_{1}, \\ u_{\mathrm{par}}(x), & x>x_{1},\end{cases}
$$

where the parameter $x_{1} \geq 0$, see Figure 8 (left). In fact, there is also the possible constant solution $u_{\mathrm{r}}(x) \equiv 3$ (corresponding to $x_{1} \rightarrow \infty$ ), see Figure 8 (right). The solution has at most two jumps. Firstly, we can conclude that all possible jumps at $x=0$, which are $\left(u_{-}, u_{+}\right)=(1,3),\left(1, u_{\alpha}\right)$ and $\left(3, u_{\alpha}\right)$, satisfy Condition $\Gamma$ with $\bar{u}=3$. Secondly, the possible jump at $x=x_{1}>0$ from 3 to $u_{\alpha}$ satisfies (4.1), which is Condition $\Gamma$ in the special case $f=g$ and $A=B$.

- $u_{\alpha} \geq 5$ : The solution is never parabolic, since this required $3<u_{\alpha}<5$. The solution on the left-hand side is either (6.5), (6.6) or (6.7), with the possibilities $u_{-}=1$ and 3 . Similarly, the solution on the right-hand side is either $u_{\mathrm{r}}(x) \equiv 3, u_{\mathrm{r}}(x) \equiv 5$, or there is a jump at $x_{1}>0$ from 3 to 5 , see Figure 7 (right). All the possible jumps $\left(u_{-}, u_{+}\right)=(1,3),(1,5)$ and $(3,5)$ satisfy Condition $\Gamma$ with $\bar{u}=3$. 

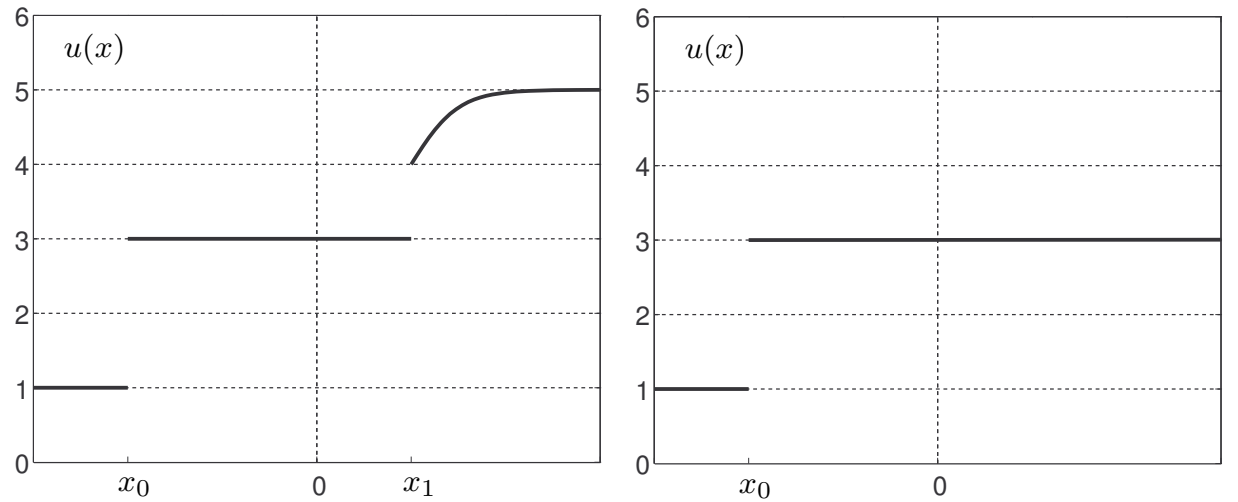

Fig. 8. Stationary solutions when $\eta=3$ and $3 \leq u_{\alpha}=4<5$. Possible locations of the discontinuities are $x_{0} \leq 0$ and $x_{1} \geq 0$. Hence, the solution with only one jump at $x=0$ from 1 to $u_{\alpha}=4$ is also possible.

\subsubsection{The constant flux is $\eta<3$}

The graphs of $f$ and $g$ reveal that the same qualitative behaviour will appear for any $\eta<3$. For simplicity, we let $\eta=0$ in this subsection.

In $x<0$, the possible solutions are the following:

$$
\begin{aligned}
& u_{1}(x) \equiv 0, \\
& u_{1}(x)= \begin{cases}0, & x<x_{0}, \\
4, & x_{0}<x<0,\end{cases} \\
& u_{1}(x) \equiv 4,
\end{aligned}
$$

for some constant $x_{0}<0$. From (6.4) we can conclude that the only possibility for a parabolic solution in a right neighbourhood of $x=x_{1} \geq 0$ is when $2<u_{\alpha}<6$. The solution of (6.3) for $2<u_{\alpha}<6$ is

$$
u_{\mathrm{par}}(x)=\frac{6 e^{4\left(x-x_{1}\right)}\left(u_{\alpha}-2\right)+2\left(6-u_{\alpha}\right)}{e^{4\left(x-x_{1}\right)}\left(u_{\alpha}-2\right)+6-u_{\alpha}}, \quad x>x_{1} .
$$

Note that it increases to 6 as $x \rightarrow \infty$. For other values of $u_{\alpha}$, the only possibility is a hyperbolic piecewise constant solution. The equation $\eta=f(u)$ yields the two solutions $u=2$ and $u=6$. Hence, we can conclude the following.

- $\underline{u_{\alpha}<2}$ : Irrespective of whether $u_{-}=0$ or $u_{-}=4$, neither a hyperbolic nor a parabolic solution is possible on the right-hand side. Hence, no stationary solution exists.

- $\underline{2 \leq u_{\alpha}<4}$ : The only possible solution on the left-hand side is (6.9). With $u_{\text {par }}$ defined by (6.12), there is a parameter family of solutions on the right- 

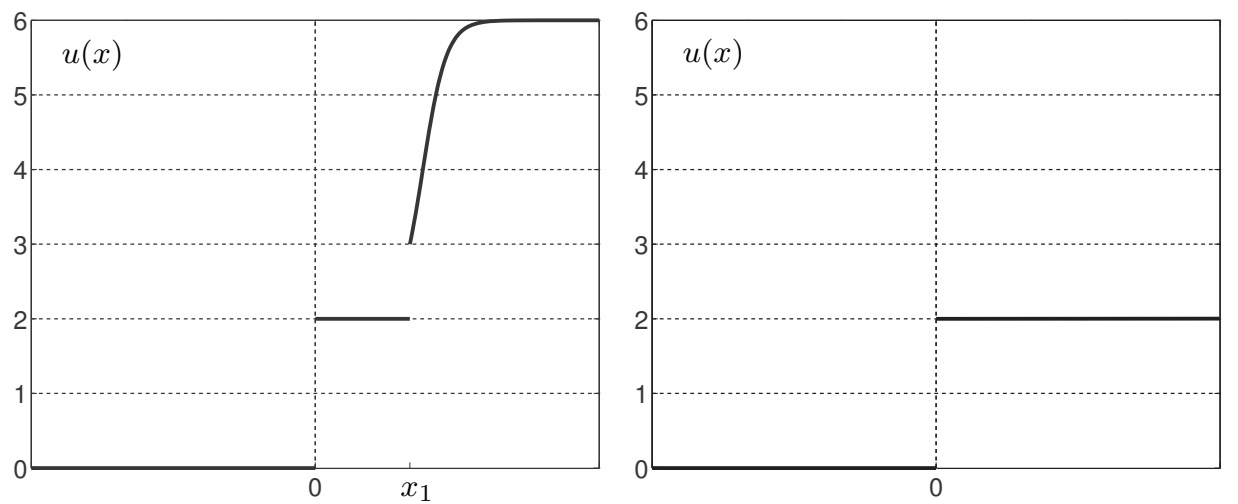

Fig. 9. Stationary solutions when $\eta=0<3$ and $2 \leq u_{\alpha}=3<4$. The discontinuity between a hyperbolic and parabolic region is located at $x_{1} \geq 0$.

hand side:

$$
u_{\mathrm{r}}(x)= \begin{cases}2, & 0<x<x_{1} \\ u_{\mathrm{par}}(x), & x>x_{1}\end{cases}
$$

where the parameter $x_{1} \geq 0$. In fact, there is also the possible constant solution $u_{\mathrm{r}}(x) \equiv 2$ (corresponding to $x_{1} \rightarrow \infty$ ), see Figure 9 . Firstly, we can conclude that the possible jumps at $x=0$, which are $\left(u_{-}, u_{+}\right)=(0,2)$ and $\left(0, u_{\alpha}\right)$, both satisfy Condition $\Gamma$ with $\bar{u}=2$. Secondly, the possible jump at $x=x_{1}>0$ from 2 to $u_{\alpha}$ satisfies the entropy inequality (4.1).

- $4 \leq u_{\alpha}<6$ : The solution on the left-hand side is either (6.9), (6.10) or (6.11). On the right-hand side the solution is (6.13) with $x_{1} \geq 0$ or $x_{1} \rightarrow \infty$, see Figure 10. The possible jumps at $x=0$, which satisfy Condition $\Gamma$, are $\left(u_{-}, u_{+}\right)=(0,2)$ with $\bar{u}=2,\left(0, u_{\alpha}\right)$ with $\bar{u}=2$, and $\left(4, u_{\alpha}\right)$ with $\bar{u}=4$. The other jumps satisfy Oleinik's entropy condition (3.9). The solution has at most two jumps.

- $\underline{u_{\alpha} \geq 6}$ : The solution is always hyperbolic. On the left-hand side it is either (6.9), (6.10) or (6.11). Similarly, on the right-hand side it is $u_{\mathrm{r}}(x) \equiv 2$, $u_{\mathrm{r}}(x) \equiv 6$ or there is a jump at $x_{1}>0$ from 2 to 6 , see Figure 11. The possible jumps that satisfy Condition $\Gamma$ are $\left(u_{-}, u_{+}\right)=(0,2)$ and $(0,6)$ with $\bar{u}=2$, and $\left(u_{-}, u_{+}\right)=(4,6)$ with $\bar{u}=4$.

\subsubsection{The constant flux satisfies $3<\eta \leq 4$}

Assume first that $3<\eta<4$. It will be obvious that the case $\eta=4$ can then be seen as a limit case. Denote the two solutions of the equation $\eta=g(u)$ by $u_{g 1}$ and $u_{g 2}>u_{g 1}$ and analogously for $f$. Hence, we have (cf. Figure 6 , left)

$$
u_{g 1}<u_{g 2}<u_{f 1}<u_{f 2} .
$$



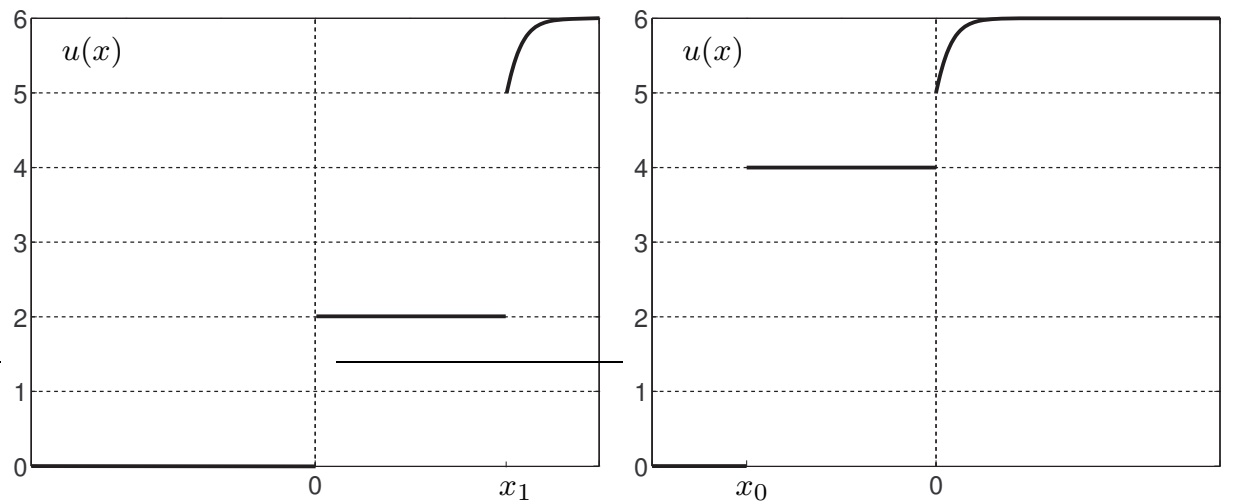

Fig. 10. Stationary solutions when $\eta=0<3$ and $4 \leq u_{\alpha}=5<6$. The discontinuities are located at $x_{1} \geq 0$ or $x_{1} \rightarrow \infty$, and $x_{0} \leq 0$ or $x_{0} \rightarrow-\infty$. Hence, the solution with only one jump from 0 to 5 is also possible.
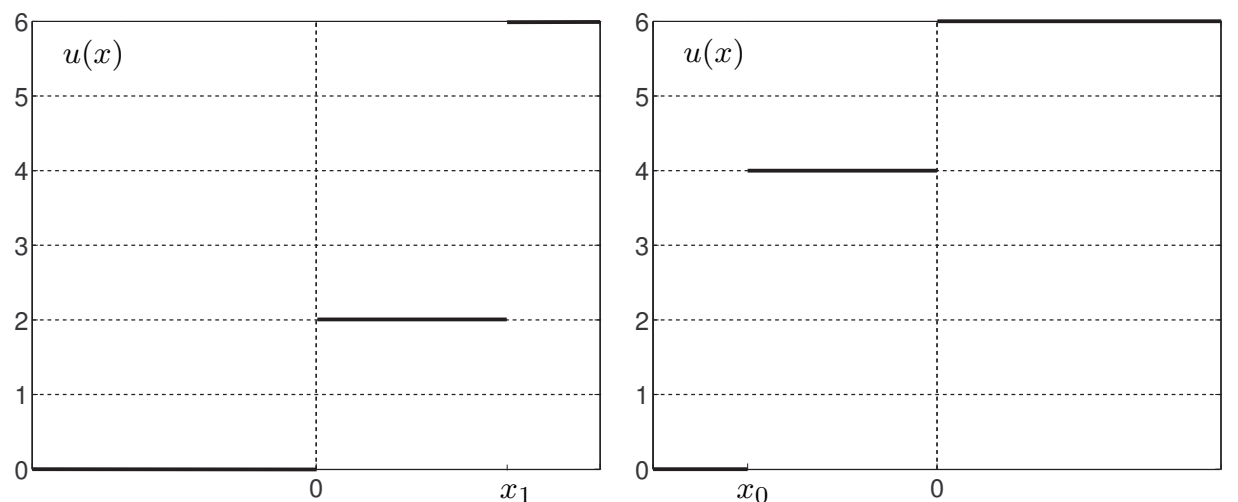

Fig. 11. Stationary solutions when $\eta=0<3$ and $u_{\alpha}=6$. The discontinuities are located at $x_{1} \geq 0$ or $x_{1} \rightarrow \infty$, and $x_{0} \leq 0$ or $x_{0} \rightarrow-\infty$. Hence, the solution with only one jump from 0 to 6 is also possible.

We can conclude, partly that either $u_{-}=u_{g 1}$ or $u_{-}=u_{g 2}$ must hold, partly that the two 'hyperbolic' possibilities in $x>0$ are $u_{f 1}$ and $u_{f 2}$, partly that the only possibility for a parabolic solution in $x>0$ is when $u_{f 1}<u_{\alpha}<u_{f 2}$, see (6.4).

If $u_{\alpha}<u_{f 1}$, then a hyperbolic solution in $x>0$ is also ruled out since such a solution has to satisfy $\max \left(u_{-}, u_{+}\right) \leq u_{\alpha}$ by Lemma 6.1 .

If $u_{\alpha} \geq u_{f 1}$, a solution with a jump from $u_{-}=u_{g 1}$ or $u_{g 2}$ up to $u_{+} \geq u_{f 1}$ is a weak solution. However, since both graphs of $f$ and $g$ lie below the flux level $\eta$ in the interval $\left(u_{g 2}, u_{f 1}\right) \subset\left[u_{-}, u_{+}\right]$, there exists no $\bar{u} \in\left[u_{-}, u_{+}\right]$to fulfil Condition $\Gamma$. Hence, no stationary solution exists. 

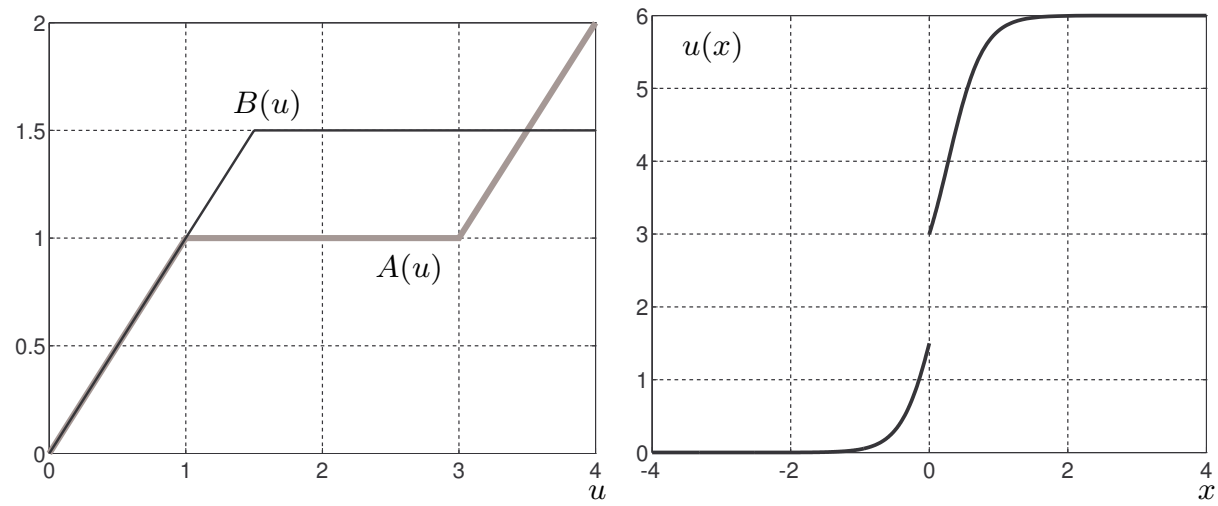

Fig. 12. The diffusion functions (left) and the parabolic solution with a jump when $\eta=0$.

\subsection{Example of a discontinuity between parabolic regions}

In order to exemplify Theorem 6.2 with parabolic regions on both sides of $x=0$, we choose the same flux functions as above, but change the diffusion functions, see Figure 12 (left):

$$
\begin{array}{ll}
f(u)=(u-2)(6-u), & A(u)=\max (\min (u, 1), u-2), \\
g(u)=u(4-u), & B(u)=\min (u, 3 / 2) .
\end{array}
$$

When $\eta=0$ a stationary solution is

$$
u(x)= \begin{cases}\frac{12}{3+5 e^{-4 x}}, & x<0, \\ \frac{6\left(e^{4 x}+1\right)}{3+e^{4 x}}, & x>0,\end{cases}
$$

see Figure 12 (right). The discontinuity satisfies Condition $\Gamma$ with $\bar{u}=u_{+}=3$.

Note that by Lemma 6.1 any solution has to satisfy $u_{-}, u_{+} \in[3 / 2,3]$, since this is the largest interval where both $A$ and $B$ are constant. Other solutions are obtained if the solution (6.14) on the right-hand side is substituted for $u_{\mathrm{r}}(x) \equiv 2$ or

$$
u_{\mathrm{r}}(x)= \begin{cases}2, & 0<x<x_{1}, \\ \frac{6\left(e^{4\left(x-x_{1}\right)}+1\right)}{3+e^{4\left(x-x_{1}\right)}}, & x>x_{1},\end{cases}
$$

for some $x_{1}>0$. Condition $\Gamma$ is satisfied with $\bar{u}=u_{+}=2$ and the jump at $x_{1}$ from 2 to 3 is satisfied by (4.1).

\subsection{Examples of non-monotone solutions}

Theorem 6.2 yields that the only possibility for a non-monotone solution in a neighbourhood of $x=0$ is a continuous one. This can be exemplified by the following 

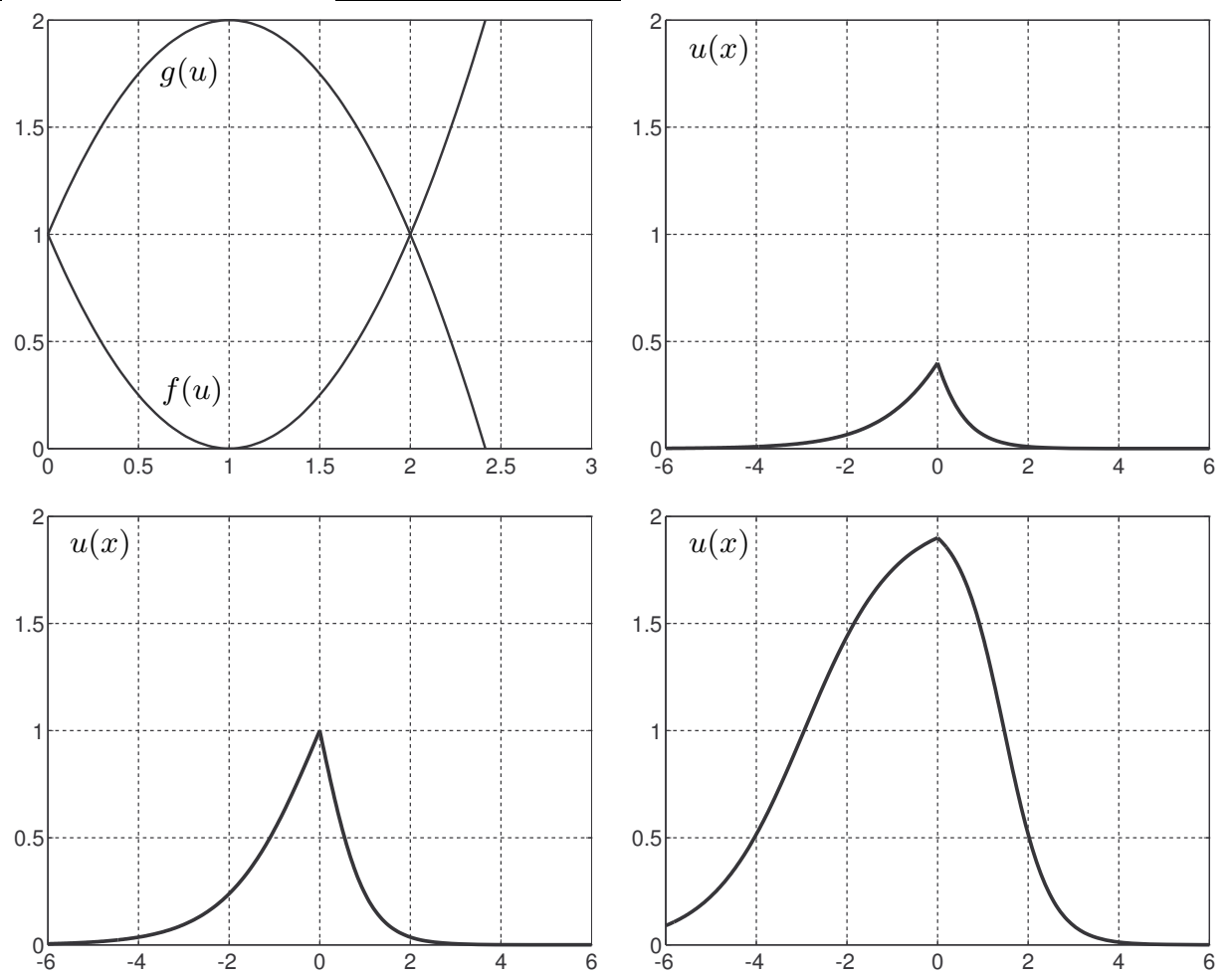

Fig. 13. The flux functions $g$ and $f$ and three examples $(a=0.4,1$ and 1.9) of the parameter family of non-monotone continuous solutions when $A(u)=u B(u)=2 u$ and $\eta=1$.

functions, see Figure 13 (upper left):

$$
\begin{array}{ll}
f(u)=(u-1)^{2}, & A(u)=u, \\
g(u)=2-(u-1)^{2}, & B(u)=b u,
\end{array}
$$

where $b>0$. Since the diffusion functions are never constant, a discontinuity is not possible, according to Lemma 6.1. Let $\eta=1$. Then the two intersections of $g$ and $f$ coincide with the solutions of the equations $\eta=g(u)$ and $\eta=f(u)$. Hence, two continuous and monotone solutions are $u(x) \equiv 0$ and $u(x) \equiv 2, x \in \mathbb{R}$. To obtain non-constant bounded solutions of (6.1) and the corresponding equation for $f$, which are pieced together continuously at $x=0$, it must hold that $0<u(0)<2$. Then the solution family is

$$
u(x)=\left\{\begin{array}{ll}
\frac{2 a}{a+(2-a) e^{-2 x / b}}, & x<0, \\
\frac{2 a}{a+(2-a) e^{2 x}}, & x>0,
\end{array} \quad \text { where } a=u(0) \in(0,2) .\right.
$$

see Figure 13. 


\section{Acknowledgements}

Raimund Bürger, University of Concepción, and Anders Holst, Lund University, are gratefully acknowledged for interesting discussions and helpful comments during the preparation of this paper. I would also like to thank the communicating editor Kenneth H. Karlsen for his invaluable suggestions. This work has been supported by the Swedish Research Council (Vetenskapsrådet), project 621-2005-3873.

\section{References}

[1] Adimurthi, S. Mishra, and G. D. Veerappa Gowda. Godunov-type methods for conservation laws with a flux function discontinuous in space. SIAM J. Numer. Anal., 42:179-208, 2004.

[2] Adimurthi, S. Mishra, and G. D. Veerappa Gowda. Optimal entropy solutions for conservation laws with discontinuous flux-functions. J. Hyperbolic Differ. Equ., 2:783$837,2005$.

[3] Adimurthi, S. Mishra, and G. D. Veerappa Gowda. Convergence of godunov type methods for a conservation law with a spatially varying discontinuous flux function. Math. Comp., 76:1219-1242, 2007.

[4] Adimurthi, S. Mishra, and G. D. Veerappa Gowda. Existence and stability of entropy solutions for a conservation law with discontinuous non-convex fluxes. Netw. Heterog. Media, 2:127-157, 2007.

[5] G. Aguilar, F. Lisbona, and M. Madaune-Tort. Analysis of a nonlinear parabolichyperbolic problem. Adv. Math. Sci. Appl., 7:165-181, 1997.

[6] E. Audusse and B. Perthame. Uniqueness for scalar conservation laws with discontinuous flux via adapted entropies. Proc. Roy. Soc. Edinburgh A, 135:253-265, 2005.

[7] F. Bachmann and J. Vovelle. Existence and uniqueness of entropy solution of scalar conservation law with a flux function involving discontinuous coefficients. Comm. Partial Differential Equations, 31:371-395, 2006.

[8] P. Baiti and H. Jenssen. Well-posedness for a class of $2 \times 2$ conservation laws with $L^{\infty}$ data. J. Differential Equations, 140:161-185, 1997.

[9] R. Bürger, A. Garcìa, K. H. Karlsen, and J. D. Towers. Difference schemes, entropy solutions, and speedup impulse for an inhomogeneous kinematic traffic flow model. To appear in Netw. Heterog. Media.

[10] R. Bürger and K. H. Karlsen. On a diffusively corrected kinematic-wave traffic model with changing road surface conditions. Math. Models Methods Appl. Sci., 13:17671799, 2003.

[11] R. Bürger, K. H. Karlsen, C. Klingenberg, and N. H. Risebro. A front tracking approach to a model of continuous sedimentation in ideal clarifier-thickener units. Nonl. Anal. Real World Appl., 4:457-481, 2003.

[12] R. Bürger, K. H. Karlsen, S. Mishra, and J. D. Towers. On conservation laws with discontinuous flux. In Y. Wang and K. Hutter, editors, Trends in Applications of Mathematics to Mechanics, pages 75-84, Aachen, 2005. Shaker Verlag.

[13] R. Bürger, K. H. Karlsen, N. H. Risebro, and J. D. Towers. On a model for continuous sedimentation in vessels with discontinuously varying cross-sectional area. In T.Y. Hou and E. Tadmor, editors, Hyperbolic Problems: Theory, Numerics, Applications. Proceedings of the Ninth International Conference on Hyperbolic Problems Held in CalTech, Pasadena, March 25-29, 2002, pages 397-406. Springer Verlag Berlin, 2003.

[14] R. Bürger, K. H. Karlsen, N. H. Risebro, and J. D. Towers. Well-posedness in $B V_{t}$ and convergence of a difference scheme for continuous sedimentation in ideal clarifier- 
thickener units. Numerische Mathematik, 97:25-65, 2004.

[15] R. Bürger, K. H. Karlsen, and J. D. Towers. A model of continuous sedimentation of flocculated suspensions in clarifier-thickener units. SIAM J. Appl. Math., 65:882-940, 2005.

[16] R. Bürger, K. H. Karlsen, and J. D. Towers. An Engquist-Osher-type scheme for conservation laws with discontinuous flux adapted to flux connections. Preprint, available at http://www.math.ntnu.no/conservation/, 2007.

[17] R. Bürger and A. Narváez. Steady-state, control, and capacity calculations for flocculated suspensions in clarifier-thickeners. International Journal of Mineral Processing, 84(1-4):274-298, 2007.

[18] S. Diehl. On scalar conservation laws with point source and discontinuous flux function. SIAM J. Math. Anal., 26(6):1425-1451, 1995.

[19] S. Diehl. A conservation law with point source and discontinuous flux function modelling continuous sedimentation. SIAM J. Appl. Math., 56(2):388-419, 1996.

[20] S. Diehl. Scalar conservation laws with discontinuous flux function: I. The viscous profile condition. Comm. Math. Phys., 176:23-44, 1996.

[21] S. Diehl. Continuous sedimentation of multi-component particles. Math. Methods Appl. Sci., 20:1345-1364, 1997.

[22] S. Diehl. Operating charts for continuous sedimentation I: Control of steady states. J. Eng. Math., 41:117-144, 2001.

[23] S. Diehl. Operating charts for continuous sedimentation II: Step responses. J. Eng. Math., 53:139-185, 2005.

[24] S. Diehl. Operating charts for continuous sedimentation III: Control of step inputs. J. Eng. Math., 54:225-259, 2006.

[25] S. Diehl. Operating charts for continuous sedimentation IV: Limitations for control of dynamic behaviour. J. Eng. Math., 60:249-264, 2008.

[26] S. Diehl. A regulator for continuous sedimentation in ideal clarifier-thickener units. J. Eng. Math., 60:265-291, 2008.

[27] S. Diehl and N.-O. Wallin. Scalar conservation laws with discontinuous flux function: II. On the stability of the viscous profiles. Comm. Math. Phys., 176:45-71, 1996.

[28] C. J. Duijn, J. Molenaar, and M. J. Neef. The effect of capillary forces on immiscible two-phase flow in heterogeneous porous media. Transp. Porous Media, 21:71-93, 1995.

[29] S. Evje and K. H. Karlsen. Monotone difference approximations of $B V$ solutions to degenerate convection-diffusion equations. SIAM J. Numer. Anal., 37:1838-1860, 2000.

[30] T. Gimse and N. H. Risebro. Riemann problems with a discontinuous flux function. In B. Engquist and B. Gustavsson, editors, Third International Conference on Hyperbolic Problems, Theory, Numerical Methods and Applications, volume I, pages 488-502, 1990.

[31] T. Gimse and N. H. Risebro. Solution of the Cauchy problem for a conservation law with a discontinuous flux function. SIAM J. Math. Anal., 23(3):635-648, 1992.

[32] J. M. Greenberg, A. Y. Leroux, R. Baraille, and A. Noussair. Analysis and approximation of conservation laws with source terms. SIAM J. Num. Anal., 34:1980-2007, 1997.

[33] E. F. Kaasschieter. Solving the Buckley-Leverett equation with gravity in a heterogeneous porous medium. Comput. Geosci., 3:23-48, 1999.

[34] K. H. Karlsen, N. H. Risebro, and J. D. Towers. Upwind difference approximations for degenerate parabolic convection-diffusion equations with a discontinuous coefficient. IMA J. Numer. Anal., 22:623-664, 2002.

[35] K. H. Karlsen, N. H. Risebro, and J. D. Towers. $L^{1}$ stability for entropy solutions of 
nonlinear degenerate parabolic convection-diffusion equations with discontinuous coefficients. Trans. Royal Norwegian Society Sci. Letters (Skr. K. Nor. Vidensk. Selsk.), 3:49, 2003.

[36] K. H. Karlsen and J. D. Towers. Convergence of the Lax-Friedrichs scheme and stability for conservation laws with a discontinuous space-time dependent flux. Chinese Ann. Math. Ser. B., Vol. 25(3):287-318, 2004.

[37] R. A. Klausen and N. H. Risebro. Stability of conservation laws with discontinuous coefficients. J. Differential Equations, 157:41-60, 1999.

[38] C. Klingenberg and N. H. Risebro. Convex conservation laws with discontinuous coefficients. Existence, uniqueness and asymptotic behavior. Comm. Partial Differential Equations, 20:1959-1990, 1995.

[39] C. Klingenberg and N. H. Risebro. Stability of a resonant system of conservation laws modeling polymer flow with gravitation. J. Differential Equations, 170:344-380, 2001.

[40] S. N. Kružkov. First order quasilinear equations in several independent variables. Math. USSR-Sb., 10:217-243, 1970.

[41] S. Mochon. An analysis of the traffic on highways with changing surface conditions. Math. Model., 9(1):1-11, 1987.

[42] J. Molenaar. Entropy conditions for heterogeneity induced shocks in two-phase flow problems. In A. P. Bourgeat, C. Carasso, S. Luckhaus, and A. Mikelic, editors, Mathematical Modelling of Flow Through Porous Media. World Scientific, Singapore, 1995.

[43] O. A. Oleinik. Uniqueness and stability of the generalized solution of the Cauchy problem for a quasi-linear equation. Uspekhi Mat. Nauk, 14:165-170, 1959. Amer. Math. Soc. Trans. Ser. 2, 33, (1964), pp. 285-290.

[44] N. Seguin and J. Vovelle. Analysis and approximation of a scalar conservation law with a flux function with discontinuous coefficients. Math. Models Methods Appl. Sci., 13:221-257, 2003.

[45] J. D. Towers. Convergence of a difference scheme for conservation laws with a discontinuous flux. SIAM J. Numer. Anal., 38:681-698, 2000.

[46] A. I. Vol'pert and S. I. Hudjaev. Cauchy's problem for degenerate second order quasilinear parabolic equations. Math. USSR Sbornik, 7:365-387, 1969.

[47] Z. Wu and J. Yin. Some properties of functions in $B V_{x}$ and their applications to the uniqueness of solutions for degenerate quasilinear parabolic equations. Northeast. Math. J., 5:395-422, 1989. 